\title{
A SURVEY ON COSYMPLECTIC GEOMETRY
}

\author{
BENIAMINO CAPPELLETTI-MONTANO, ANTONIO DE NICOLA, AND IVAN YUDIN
}

\begin{abstract}
We give an up-to-date overview of geometric and topological properties of cosymplectic and coKähler manifolds. We also mention some of their applications to time-dependent mechanics.
\end{abstract}

\section{CONTEnTs}

1. Introduction

2. Cosymplectic structures

2.1. Basic definitions and properties

2.2. Reduction of cosymplectic structures

2.3. Universal model for cosymplectic manifolds

2.4. Canonical homology

3. CoKähler structures

3.1. Basic definitions and properties

3.2. Examples of coKähler manifolds

3.3. CoKähler manifolds and Kähler geometry

3.4. CoKähler structures on hypersurfaces of Kähler manifolds

3.5. Homogeneous coKähler manifolds

3.6. Curvature of coKähler manifolds

3.7. Topology of compact coKähler manifolds

3.8. Coeffective cohomology

3.9. Rational homotopy type

3.10. Cosymplectic 3-structures 2

4. Further topics

4.1. Submanifolds of coKähler manifolds

4.2. Harmonic maps and coKähler geometry

4.3. Generalizations of cosymplectic and coKähler manifolds

4.4. Almost coKähler manifolds satisfying further conditions References

2000 Mathematics Subject Classification. Primary 53D15, Secondary 53C15, 53D17.

Key words and phrases. cosymplectic; coKähler; 3-structure; reduction; Poisson; time-dependent; Hamiltonian; almost contact; cosymplectic space-forms; nullity distribution; mapping torus; coeffective cohomology; formality; harmonic map; locally conformal; para-cosymplectic; para-coKähler; k-cosymplectic; generalized almost contact; nearly cosymplectic; nearly coKähler; Einstein-Weyl; conformally flat.

Research partially supported by CMUC, funded by the European program COMPETE/FEDER, by FCT (Portugal) grants PEst-C/MAT/UI0324/2011, PTDC/MAT/099880/2008 (A.D.N.), SFRH/BPD/31788/2006 (I.Y.), by MICINN (Spain) grant MTM2009-13383 (A.D.N.), and by Prin 2010/11 - Varietà reali e complesse: geometria, topologia e analisi armonica - Italy (B.C.M.). 


\section{INTRODUCTION}

In recent years there has been an increasing interest towards almost contact geometry and related topics, both from the pure geometrical point of view and due to its applications in wide areas of Physics. In particular, the odd dimensional manifolds proved to be very important for some physical theories like supergravity and M-theory.

An important class of almost contact manifolds is given by cosymplectic manifolds. By a cosymplectic manifold in this paper we mean a smooth manifold of dimension $2 n+1$ endowed with a closed 1 -form $\eta$ and a closed 2 -form $\omega$ such that $\eta \wedge \omega^{n}$ is a volume form. This is the original definition of cosymplectic manifold, due to Libermann and dating back to 1958. Later Blair used the term "cosymplectic" with a different meaning, namely for denoting cosymplectic manifolds endowed with a compatible almost contact metric structure satisfying a normality condition. Despite the wide use of Blair's terminology, we will call the latter manifolds coKähler, which is becoming a common practice now.

Also, there is a weaker notion of almost coKähler manifold (called sometimes almost cosymplectic!) which is just a cosymplectic manifold with a compatible almost contact metric structure, without the normality condition. Note that every cosymplectic manifold admits an almost coKähler structure.

Since the foundational papers of Libermann and Blair, there have been many advances in the subject and its related topics. Several papers dealing with cosymplectic or coKähler manifolds appeared in the last 40 years, even though in a rather sporadic way. Unfortunately, there is neither a monograph nor a paper which gives an exposition of the state of art of the theory. In fact the recent very well-written monographs on contact geometry - the Blair's and Boyer-Galicki's books [15], [23] - treat, from different points of view, just contact metric manifolds (including Sasakian manifolds), leaving for cosymplectic geometry not much space.

We hope that the present survey can help to fill this gap and to give a unified view of the subject.

The importance of cosymplectic manifolds for the geometric description of time-dependent mechanics is nowadays widely recognized. In most of the numerous formulations of time-dependent mechanics cosymplectic manifolds do play a major role: either in the classical descriptions of regular Lagrangian systems (see e.g. 54]) and of Hamiltonian systems [54, 106], or in the more elegant descriptions á la Tulczyjew ([55, 93]) in terms of Lagrangian submanifolds. It is far outside the scope of our presentation to make a detailed review of these approaches or even a complete list of papers. The 1991 paper 64 contains a comparative review at that time of various equivalent approaches to the geometric treatment of regular time-dependent Hamiltonian systems. For further information about more recent literature on the geometric formulations of time-dependent mechanics see [7, 54, 106] and references therein. Here we just briefly summarize, as an example, how cosymplectic manifolds appear in the geometric setup of time-dependent Lagrangian mechanics [7, 54. In such a model the configuration manifold of autonomous Lagrangian mechanics is replaced by a fibration $\pi: M \rightarrow \mathbb{R}$ and the jet bundle $J^{1} \pi$ of the local sections of $\pi$ is the velocity phase space of the system. In the simplest case the fibration is trivial, i.e. $M \cong Q \times \mathbb{R}$ where $Q$ is a manifold, and one has $J^{1} \pi \cong T M \times \mathbb{R}$. In general the dynamics is controlled by a Lagrangian density $\mathcal{L} \in \Omega^{1}\left(J^{1} \pi\right)$ which is a semibasic 1-form. The Poincaré-Cartan forms associated with the Lagrangian density $\mathcal{L}$ are defined using the "vertical endomorphism" $S$ of the bundle $J^{1} \pi$ (see 64, 136]),

$$
\Theta_{\mathcal{L}}=i_{S} \mathrm{~d} \mathcal{L}+\mathcal{L}, \quad \Omega_{\mathcal{L}}=-\mathrm{d} \Theta_{\mathcal{L}}
$$

Geometrically, the Lagrangian density is regular if and only if the pair $\left(\mathrm{d} t, \Omega_{\mathcal{L}}\right)$ is a cosymplectic structure on the manifold $J^{1} \pi$, where by $\mathrm{d} t$ we denote (with a slight abuse of notation) the pull-back on $J^{1} \pi$ of the canonical volume 1 -form of $\mathbb{R}$. Then, in the regular case, the dynamics of the system is controlled by the integral curves of the Reeb vector field $\xi_{\mathcal{L}}$. In the singular case, the structure $\left(\mathrm{d} t, \Omega_{\mathcal{L}}\right)$ is not cosymplectic and the dynamics is controlled by an implicit differential equation, not necessarily integrable.

We devote the first part of the survey to the basic properties of cosymplectic structures. An important role in the theory is played by the reduction theory, which was developed by Albert in [1] in order to 
be able to consider the reduction of time-dependent Hamiltonian systems with symmetry groups. The reduction procedure was extended to the singular case by de León and Tuynman [60. Then, in [26] the Albert reduction theorem was adapted to deal with the case of a time-dependent mechanical system with constraints.

As we pointed out before, any cosymplectic manifold can be endowed with a Riemannian metric which makes it an almost coKähler manifold. We thus study, in the second part of the paper, the geometry of almost coKähler manifolds. We collect the most important results of the theory and the various ways for constructing explicit examples (by suspensions, by using Lie groups, as hypersurfaces of Kähler manifolds, etc). Due to the importance of the subject, a large part is dedicated to the study of the curvature properties of almost coKähler manifolds and to the topology of cosymplectic and coKähler manifolds. This last topic has become of great interest in last years, after the very remarkable work 40, of Chinea, de León, Marrero on the topology of compact coKähler manifolds. Recently Li ([107]) and then Bazzoni and Oprea [9] discovered new methods for studying the topology of coKähler manifolds, which allow us to re-obtain many of the Chinea - de León - Marrero's results.

We also try to explain the several interplays with Kähler geometry, which make coKähler manifolds the most natural odd-dimensional counterpart of Kähler manifolds. This becomes even more evident when one passes to the setting of 3-structures. Indeed, while both coKähler and Sasakian manifolds admit a transversal Kähler structure, at the level of 3-structures only the former admit a transversal hyper-Kähler structure.

There are many further topics related to cosymplectic geometry, that have found almost no room in this survey due to the lack of space. We mention only few of them: the study of submanifolds of cosymplectic / coKähler manifolds, the theory of harmonic maps, Einstein-Weyl structures, and several remarkable generalizations such as locally conformal cosymplectic manifolds, $k$-cosymplectic manifolds or generalized almost contact structures. All these subjects, which in turn often have relations with each other and with Physics, show how rich and wide is the field which we call "cosymplectic geometry".

Acknowledgement. The authors thank the anonymous referees for the careful reading and for having improved the paper.

\section{Cosymplectic Structures}

2.1. Basic definitions and properties. Cosymplectic manifolds were introduced by Libermann (see [108, 109]).

Definition 2.1. An almost cosymplectic structure on a manifold $M$ of odd dimension $2 n+1$ is a pair $(\eta, \omega)$, where $\eta$ is a 1 -form and $\omega$ is a 2 -form such that $\eta \wedge \omega^{n}$ is a volume form on $M$. The structure is said to be cosymplectic if $\eta$ and $\omega$ are closed.

If $(\eta, \omega)$ is an almost cosymplectic structure on $M$, the triple $(M, \eta, \omega)$ is said to be an almost cosymplectic manifold. Similarly, a cosymplectic manifold is a manifold $M$ endowed with a cosymplectic structure $(\eta, \omega)$. In the language of $G$-structures, an almost cosymplectic structure can be defined equivalently as an $1 \times S p(n, \mathbb{R})$-structure.

Among the early studies of cosymplectic manifolds, we mention those of Lichnerowicz [11, 112, $\mathrm{Br}$ uschi [24, Takizawa [140] and Okumura 124]. Lichnerowicz studied the Lie algebra of infinitesimal automorphisms of a cosymplectic manifold, in analogy with the symplectic case. Bruschi investigated homogeneous cosymplectic spaces. Takizawa reviewed the Lagrange brackets and the infinitesimal transformations. The work of Okumura will be described in the next section since it actually belongs to the coKähler domain. 
Every almost cosymplectic structure $(\eta, \omega)$ on $M$ induces an isomorphism of $C^{\infty}(M)$-modules $b_{(\eta, \omega)}$ : $\mathfrak{X}(M) \rightarrow \Omega^{1}(M)$ defined by

$$
b_{(\eta, \omega)}(X)=i_{X} \omega+\eta(X) \eta,
$$

for every vector field $X \in \mathfrak{X}(M)$. A vector bundle isomorphism (denoted with the same symbol) $b_{(\eta, \omega)}$ : $T M \rightarrow T^{*} M$ is also induced. Then the vector field $\xi=b_{(\eta, \omega)}^{-1}(\eta)$ on $M$ is called the Reeb vector field of the almost cosymplectic manifold $(M, \eta, \omega)$ and is characterized by the following conditions

$$
i_{\xi} \omega=0, \quad \eta(\xi)=1 .
$$

Conversely, we have the following characterization of almost cosymplectic manifolds that follows from [1. Proposition 2].

Proposition 2.2. Let $M$ be a manifold endowed with a 1-form $\eta$ and a 2-form $\omega$ such that the map $b_{(\eta, \omega)}: \mathfrak{X}(M) \rightarrow \Omega^{1}(M)$ defined by (2.1) is an isomorphism. Assume also that there exists a vector field $\xi$ such that $i_{\xi} \omega=0$ and $\eta(\xi)=1$. Then, $M$ has odd dimension and $(M, \eta, \omega)$ is an almost cosymplectic manifold with Reeb vector field $\xi$.

By means of the isomorphism $b_{(\eta, \omega)}: \mathfrak{X}(M) \rightarrow \Omega^{1}(M)$ one can associate with every function $f \in$ $C^{\infty}(M)$ the vector field grad $f \in \mathfrak{X}(M)$, called gradient vector field, which is defined by

$$
\operatorname{grad} f=b_{(\eta, \omega)}^{-1}(\mathrm{~d} f) \text {. }
$$

Equivalently one has (cf. [1])

$$
i_{\operatorname{grad} f} \omega=\mathrm{d} f-\xi(f) \eta, \quad \eta(\operatorname{grad} f)=\xi(f) .
$$

If the manifold $M$ is cosymplectic, then it is easy to check that the bracket defined on $C^{\infty}(M)$ by

$$
\{f, g\}=\omega(\operatorname{grad} f, \operatorname{grad} g),
$$

is a Poisson bracket. Thus, every cosymplectic manifold $(M, \eta, \omega)$ has an induced Poisson structure. Its Poisson bivector field is defined by

$$
\pi_{(\eta, \omega)}(\alpha, \beta)=\omega\left(b_{(\eta, \omega)}^{-1} \alpha, b_{(\eta, \omega)}^{-1} \beta\right),
$$

for each $\alpha, \beta \in \Omega^{1}(M)$. Its symplectic leaves are precisely the leaves of the integrable distribution ker $\eta$. For further details and properties of the gradient vector fields we refer to [27].

With every function $f \in C^{\infty}(M)$ one can also associate a Hamiltonian vector field $X_{f}$ according to

$$
X_{f}=b_{(\eta, \omega)}^{-1}(\mathrm{~d} f-\xi(f) \eta)
$$

or equivalently

$$
i_{X_{f}} \omega=\mathrm{d} f-\xi(f) \eta, \quad \eta\left(X_{f}\right)=0 .
$$

One can show (see [1]) that the map $C^{\infty}(M) \rightarrow \mathfrak{X}(M)$ defined by $f \mapsto X_{f}$ is a Lie algebra antihomomorphism with respect to the Poisson bracket (2.3) and the commutator of vector fields, i.e.

$$
X_{\{f, g\}}=-\left[X_{f}, X_{g}\right] .
$$

From (2.2) and (2.5) we get easily that

$$
i_{X_{f}} \omega=i_{\operatorname{grad} f} \omega
$$

and $X_{f}=\operatorname{grad} f$ if and only if $\xi(f)=0$. Thus, the Poisson structure of a cosymplectic manifold $(M, \eta, \omega)$ can be equivalently written as

$$
\{f, g\}=\omega\left(X_{f}, X_{g}\right) .
$$


Any cosymplectic manifold $(M, \eta, \omega)$ of dimension $2 n+1$ admits around any point local coordinates $\left(t, q^{i}, p_{i}\right), i=1, \ldots, n$, such that

$$
\omega=\sum_{i=1}^{n} \mathrm{~d} q^{i} \wedge \mathrm{d} p_{i}, \quad \eta=\mathrm{d} t, \quad \xi=\frac{\partial}{\partial t} .
$$

This fact is usually referred to as Darboux theorem.

Cosymplectic manifolds can be thought of as an odd-dimensional counterpart of symplectic manifolds. In fact, on any cosymplectic manifold $(M, \eta, \omega)$ the so-called horizontal distribution ker $\eta$ is integrable to a symplectic foliation of codimension 1 . On the other hand, one has the following result on the product $M \times \mathbb{R}$.

Proposition 2.3 ([59]). Let $M$ be a manifold and $\eta, \omega$ two differential forms on $M$ with degrees 1 and 2 respectively. Consider on $\tilde{M}=M \times \mathbb{R}$ the differential 2 -form $\tilde{\omega}=p^{*} \omega+p^{*} \eta \wedge \mathrm{d} t$, where $t$ is the coordinate function on $\mathbb{R}$ and $p: \tilde{M} \rightarrow M$ is the canonical projection. Then

(a) $(M, \eta, \omega)$ is a cosymplectic manifold if and only if $(\tilde{M}, \tilde{\omega})$ is a symplectic manifold.

(b) In such a case, $p$ is a Poisson morphism.

Remark 2.4. As in symplectic geometry, at any given point $p$ of a cosymplectic manifold $M$ we can define, following [60], a cosymplectic orthogonal complement of a vector subspace $E \subset T_{p} M$ by

$$
E^{\perp}:=\left\{X \in T_{p} M \mid \eta(X)=0, i_{X} \omega \in E^{o}\right\},
$$

where $E^{o} \subset T_{p}^{*} M$ is the annihilator of $E$. Then, we say that a submanifold $C \subset M$ is a coisotropic submanifold of the cosymplectic manifold $(M, \eta, \omega)$ if

(i) the Reeb vector field $\xi$ is tangent to $C$;

(ii) $(T C)^{\perp} \subset T C$.

The notion of a coisotropic submanifold $C$ of a cosymplectic manifold $M$ can be related to the usual notion of a coisotropic submanifold of a symplectic manifold in the following way. If $(M, \eta, \omega)$ is cosymplectic, then the manifold $\tilde{M}=M \times \mathbb{R}$ has a symplectic structure defined by Proposition 2.3. One then can prove that $C \subset M$ is coisotropic if and only if one has $(T(C \times \mathbb{R}))^{\perp} \subset T C \oplus\{0\}$, where $(T(C \times \mathbb{R}))^{\perp}$ denotes the symplectic orthogonal complement. It follows that $C \times \mathbb{R}$ is a special kind of coisotropic submanifold of $M \times \mathbb{R}$ in the symplectic sense.

Definition 2.5. A smooth map $\psi: M \rightarrow N$ between two cosymplectic manifolds $(M, \eta, \omega)$ and $\left(N, \eta^{\prime}, \omega^{\prime}\right)$ is said to be cosymplectic if $\psi^{*} \eta^{\prime}=\eta$ and $\psi^{*} \omega^{\prime}=\omega$. In such a case, the Reeb vector field $\xi$ of $M$ is $\psi$-projectable and its projection is the Reeb vector field $\xi^{\prime}$ of $N$, that is,

$$
T \psi \circ \xi=\xi^{\prime} \circ \psi \text {. }
$$

As in the symplectic case, a cosymplectic map is not, in general, a Poisson map. In a cosymplectic manifold $(M, \eta, \omega)$ one can use the vector bundle isomorphism $b_{(\eta, \omega)}: T M \rightarrow T^{*} M$ to pull back the canonical symplectic 2 -form $\Omega_{M}$ of the cotangent bundle $T^{*} M$ to the tangent bundle $T M$ by setting

$$
\Omega_{o}:=b_{(\eta, \omega)}^{*} \Omega_{M} .
$$

Therefore the tangent bundle of a cosymplectic manifold is a symplectic manifold. Actually, the existence of the vector bundle isomorphism $b_{(\eta, \omega)}: T M \rightarrow T^{*} M$ means that the tangent bundle of a cosymplectic manifold has a Liouville structure (also known as special symplectic structure) in the sense of Tulczyjew [146]. In [27] the authors obtain an explicit expression for the symplectic structure $\Omega_{o}$ on the tangent bundle in terms of the cosymplectic structure $(\eta, \omega)$, by using the notion of tangent derivation, again due to Tulczyjew (see e.g. [145). 
2.2. Reduction of cosymplectic structures. An action $\phi: G \times M \rightarrow M$ of a Lie group $G$ on a cosymplectic manifold $(M, \eta, \omega)$ is said to be cosymplectic if $\phi_{g}: M \rightarrow M$ is a cosymplectic map for any $g \in G$. In such a case, a smooth map $J: M \rightarrow \mathfrak{g}^{*}$ from $M$ to the dual space $\mathfrak{g}^{*}$ of the Lie algebra $\mathfrak{g}$ of $G$ is said to be a momentum map if the infinitesimal generator $a_{M} \in \mathfrak{X}(M)$ of the action associated with any $a \in \mathfrak{g}$ is the Hamiltonian vector field of the function $J_{a}: M \rightarrow \mathbb{R}$ defined by the natural pointwise pairing. Moreover, $J$ is said to be $\mathrm{Ad}^{*}$-equivariant if it is equivariant with respect to the action $\phi$ and to the coadjoint action $\mathrm{Ad}^{*}: G \times \mathfrak{g}^{*} \rightarrow \mathfrak{g}^{*}$, that is

$$
J\left(\phi_{g}(x)\right)=\operatorname{Ad}_{g^{-1}}^{*}(J(x)), \quad \text { for any } x \in M .
$$

An element $\nu \in \mathfrak{g}^{*}$ is said to be a weakly regular value of $J$ if $J^{-1}(\nu)$ is a closed submanifold of $M$ and for each $x \in J^{-1}(\nu)$ the tangent space $T_{x}\left(J^{-1}(\nu)\right)$ coincides with the kernel of $T_{x} J$. For example, if $\nu \in \mathfrak{g}^{*}$ is a regular value of $J$ (i.e. if $T_{x} J$ has full rank for every $x \in J^{-1}(\nu)$ ), then it is a weakly regular value. Note that, if $\phi: G \times M \rightarrow M$ is a free and proper action, and $J: M \rightarrow \mathfrak{g}^{*}$ is a momentum map for $\phi$, then one can prove (cf. [3]) that $J$ is a submersion, i.e. all elements $\nu \in \mathfrak{g}^{*}$ are regular values.

Let $\nu \in \mathfrak{g}^{*}$ be a weakly regular value of an $\operatorname{Ad}^{*}$-equivariant momentum map $J: M \rightarrow \mathfrak{g}^{*}$ for an action $\phi: G \times M \rightarrow M$. If $G_{\nu}$ denotes the isotropy group of $\nu$ with respect to the coadjoint action, i.e.

$$
G_{\nu}=\left\{g \in G: \operatorname{Ad}_{g}^{*} \nu=\nu\right\},
$$

then $\phi$ induces an action

$$
\phi: G_{\nu} \times J^{-1}(\nu) \rightarrow J^{-1}(\nu)
$$

of $G_{\nu}$ on the submanifold $J^{-1}(\nu)$. Following Libermann and Marle [110] we will say that this action is simple (Albert calls such an action "quotientant") if the orbit space $M_{\nu}=J^{-1}(\nu) / G_{\nu}$ admits a smooth manifold structure and the canonical projection $\pi_{\nu}: J^{-1}(\nu) \rightarrow M_{\nu}$ is a surjective submersion. A case in which this happens is when the action is free and proper. If the action $\phi: G \times M \rightarrow M$ is free and proper, then the induced action of $G_{\nu}$ on $J^{-1}(\nu)$ is again free and proper, for each $\nu \in \mathfrak{g}^{*}$.

One has the following reduction theorem that yields an analogue of the well-known reduction theorem for symplectic manifolds [116].

Theorem 2.6 (Cosymplectic reduction Theorem, [1]). Let $\phi: G \times M \rightarrow M$ be a cosymplectic action of a Lie group $G$ on a cosymplectic manifold $(M, \eta, \omega)$. Suppose that $J: M \rightarrow \mathfrak{g}^{*}$ is an $\mathrm{Ad}^{*}$-equivariant momentum map associated with $\phi$ such that

$$
\xi\left(J_{a}\right)=0, \quad \text { for any } a \in \mathfrak{g},
$$

where $\xi$ is the Reeb vector field of $M$. Let $\nu \in \mathfrak{g}^{*}$ be a weakly regular value of $J$ such that the induced action of $G_{\nu}$ on $J^{-1}(\nu)$ is simple. Then, $M_{\nu}=J^{-1}(\nu) / G_{\nu}$ is a cosymplectic manifold with cosymplectic structure $\left(\eta_{\nu}, \omega_{\nu}\right)$ characterized by

$$
\pi_{\nu}^{*} \eta_{\nu}=i_{\nu}^{*} \eta, \quad \pi_{\nu}^{*} \omega_{\nu}=i_{\nu}^{*} \omega
$$

where $\pi_{\nu}: J^{-1}(\nu) \rightarrow M_{\nu}$ is the canonical projection and $i_{\nu}: J^{-1}(\nu) \hookrightarrow M$ is the canonical inclusion.

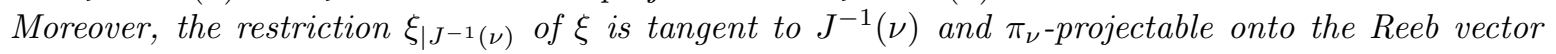
field $\xi_{\nu}$ of $M_{\nu}$.

Since each cosymplectic manifold is a Poisson manifold, the above reduction problem can be enclosed in the more general case of the reduction of Poisson structures, studied by Marsden-Ratiu [117. The supplementary information added by Albert consists in the cosymplectic nature of the reduced manifold. Furthermore, as it has been remarked in [106, one can prove that the Poisson structure induced by $\left(\eta_{\nu}, \omega_{\nu}\right)$ coincides with the reduced Poisson structure deduced by the Marsden-Ratiu reduction theorem for Poisson manifolds.

In [59] de León and Saralegi showed that the Albert reduction theorem can be obtained in a different way, by first extending the phase space $M$ according to Proposition 2.3 and then applying the MarsdenWeinstein reduction procedure for symplectic manifolds [116]. This allows them to study cosymplectic 
singular reduction, that is the reduction for singular values of the momentum map, by applying the results of Sjamaar and Lerman [137] for singular symplectic reduction. In this way, the authors of [59] are able to obtain a stratified cosymplectic space as a result of the reduction of a cosymplectic manifold with a cosymplectic action of a compact group and a momentum map.

2.3. Universal model for cosymplectic manifolds. The following more general kind of reduction of cosymplectic manifolds was considered by de León and Tuynman 60.

Definition 2.7. Let $(M, \eta, \omega)$ be a cosymplectic manifold and let $C$ be a submanifold of $M$. Suppose furthermore that the following three conditions are satisfied:

(i) the Reeb vector field $\xi$ is tangent to the submanifold $C$;

(ii) the characteristic distribution $\mathcal{F}:=\left.\left.\operatorname{ker} \eta\right|_{C} \cap \operatorname{ker} \omega\right|_{C}$ has constant rank and hence it is a regular foliation on $C$;

(iii) the foliation $\mathcal{F}$ is simple, i.e. the space of leaves $M_{r}:=C / \mathcal{F}$ has a structure of a manifold and the canonical projection $\pi: C \rightarrow M_{r}$ is a submersion.

Under the above hypotheses, it can be shown that there exist unique closed forms $\eta_{r} \in \Omega^{1}\left(M_{r}\right)$ and $\omega_{r} \in \Omega^{2}\left(M_{r}\right)$ such that $\left(M_{r}, \eta_{r}, \omega_{r}\right)$ is a cosymplectic manifold, the pullbacks of $\pi^{*} \eta_{r}$ and $\pi^{*} \omega_{r}$ coincide with the restrictions $\left.\eta\right|_{C}$ and $\left.\omega\right|_{C}$, respectively, and the Reeb vector field $\xi$ of $M$ projects onto the Reeb vector field $\xi_{r}$ of the cosymplectic manifold $M_{r}$.

In these circumstances we will say that $M_{r}$ is the reduction of $M$ by $C$.

Some properties of the cosymplectic reduction are collected in the following proposition.

Proposition $2.8(\underline{60})$. Let $\left(M_{r}, \eta_{r}, \omega_{r}\right)$ be the reduction of a cosymplectic manifold $(M, \eta, \omega)$ by a submanifold $C \subset M$. Then, $\eta$ is exact implies that $\eta_{r}$ is also exact. If the Reeb vector field $\xi$ is complete and $C$ is closed in $M$, then $\xi_{r}$ is also complete.

One could wonder if the local models for cosymplectic manifolds, which are given by the Darboux coordinates (2.8) on $\mathbb{R}^{2 n+1}$, could be general universal models, in the sense that all cosymplectic manifolds can be obtained from an $\mathbb{R}^{2 N+1}$ by reduction with respect to a submanifold. Indeed in the Darboux local model the 1-form is exact and the Reeb vector field is complete. Since for a general cosymplectic manifold the 1-form $\eta$ need not be exact, Proposition 2.8 shows that local models cannot be universal. Moreover, since the Reeb vector field of a general cosymplectic manifold need not be complete, it also follows from Proposition 2.8 that we cannot always take the submanifold $C$ to be closed. This is in sharp contrast with the symplectic case, studied in 91, in which the local model $\mathbb{R}^{2 N}$ is at the same time the universal model, and in which the reduction can always be done by a closed submanifold. The universal model for cosymplectic manifolds found by de León and Tuynman turns out to be slightly more complicated.

Let us say that a manifold is of cohomologically finite type if the de Rham cohomology $H^{k}(M)$ is a finitely generated vector space for all $k$. For any 1 -form $\eta$ on a manifold $M$ we define $\operatorname{rank}_{\mathbb{Q}}(\eta)$ by

$$
\operatorname{rank}_{\mathbb{Q}}(\eta)=\operatorname{dim}_{\mathbb{Q}}(\mathbb{Q} \cdot \operatorname{Periods}(\eta)),
$$

where

$$
\operatorname{Periods}(\eta)=\left\{\int_{z} \eta \mid z \text { a chain in } M\right\} \subset \mathbb{R} .
$$

Note that for a manifold $M$ of cohomologically finite type the $\mathbb{Q}$-dimension of $\mathbb{Q} \cdot \operatorname{Periods}(\eta)$ is always finite. We can now state the following theorem which gives the universal model for cosymplectic manifolds.

Theorem 2.9 (60]). Let $(M, \eta, \omega)$ be a cosymplectic manifold of cohomologically finite type. Then there exist integers $N$ and $k$ and real numbers $\mu_{1}, \ldots, \mu_{k}$ that are independent over $\mathbb{Q}$ such that $M$ is the reduction of the cosymplectic manifold $\left(M_{u}, \eta_{u}, \omega_{u}\right)$ by some submanifold $C \subset M_{u}$, where

$$
M_{u}=\mathbb{R} \times T^{*}\left(\mathbb{T}^{k} \times \mathbb{R}^{N}\right), \quad \eta_{u}=\mathrm{d} \theta_{\mathbb{T}^{k} \times \mathbb{R}^{N}}, \quad \omega_{u}=\mathrm{d} t+\sum_{i=1}^{k} \mu_{i} \mathrm{~d} \varphi_{i},
$$


where $\theta_{\mathbb{T}^{k} \times \mathbb{R}^{N}}$ is the Liouville 1-form, $\varphi_{i}$ are the angle coordinates on the torus, $t$ is the canonical coordinate on $\mathbb{R}$, and $k=\operatorname{rank}_{\mathbb{Q}}(\eta)$.

De León and Tuynman also proved a corresponding equivariant version of the above theorem, for which we refer to 60.

2.4. Canonical homology. The canonical homology was introduced by Koszul in 104 for any Poisson manifold. As every cosymplectic manifold can be considered as a Poisson manifold as well, it is interesting to study the properties of the canonical homology for them.

Let $M$ be a Poisson manifold and $\pi$ the Poisson bivector. Then we can define the map $\delta: \Omega^{p}(M) \rightarrow$ $\Omega^{p-1}(M)$ by $\delta=\left[i_{\pi}, d\right]$, where $i_{\pi}$ is the operator of contraction with the bivector $\pi$. It is easy to check that $\delta^{2}=0$. Therefore $\left(\Omega^{p}(M), \delta\right)$ is a complex, whose homology $H_{p}^{\text {can }}(M)$ is called canonical homology of $M$.

In the case $M^{2 n}$ is a compact symplectic manifold it was shown in [25] that $H_{p}^{c a n}(M) \cong H^{2 n-p}(M)$ for $0 \leq p \leq n$.

It was discovered in [77] that a similar result for a compact cosymplectic manifold $M^{2 n+1}$ is false by constructing an explicit counterexample of dimension 5. Nevertheless, in [77] Fernández et al. were able to construct a spectral sequence whose first page is given by $H_{p}^{c a n}(M)$ and that converges to $H^{2 n+1-p}(M)$. In particular, for any compact cosymplectic manifold $M$

$$
\operatorname{dim} H_{p}^{c a n}(M) \geq b_{2 n+1-p}(M), \quad 0 \leq p \leq 2 n+1 .
$$

Further topological properties of cosymplectic manifolds will be reviewed in the almost coKähler context in Sections 3.7 and 3.8 .

\section{CoKähler Structures}

Historically coKähler manifolds were defined by Blair in [12 in the context of quasi-Sasakian manifolds and, independently, by Ogiue in 123 as normal almost contact metric manifolds such that the fundamental 2-form $\Phi$ and the 1-form $\eta$ are closed. Actually the notion was used, even if implicitly, for the first time in an article of Okumura (124), where he studied the hypersurfaces of a Kähler manifold of constant holomorphic sectional curvature. Okumura studied the conditions for which such a hypersurface carries a cosymplectic structure, but he did not realize that in fact he was dealing with a general geometric structure richer than the cosymplectic one.

It should be remarked that Ogiue used the word "cocomplex", whereas Blair the word "cosymplectic". The latter has been used in many subsequent papers and it is now widely spread across the literature. However, since it could be confusing, as the same term was used by Libermann for a different (although related) geometric structure, here we prefer the word "coKähler". This name was firstly used by Bejancu and Smaranda ([11]) and, later on, by Janssens, Vanecke ([98]) and Marrero ([114]). More recently the paper of $\operatorname{Li}([107])$ and some other subsequent papers have adopted this terminology.

3.1. Basic definitions and properties. An almost contact structure on a manifold $M$ is given by a triple $(\phi, \xi, \eta)$, where $\xi$ is a vector field, $\eta$ is a 1 -form and $\phi$ is a $(1,1)$-tensor field related by the following conditions

$$
\phi^{2}=-I+\eta \otimes \xi, \quad \eta(\xi)=1,
$$

where $I$ denotes the identity mapping. From the definition it follows that $M$ has necessarily odd dimension, say $2 n+1$. Further consequences are that $\phi \xi=0$ and $\eta \circ \phi=0$ (see [15] for the proof and any detail). This notion was introduced in 1961 by Sasaki (134). In the same year Sasaki and Hakateyama (135) introduced the odd-dimensional counterpart of the notion of complex manifold. Their idea was to 
consider the product $M \times \mathbb{R}$ of an almost contact manifold with the real line and to define on it a tensor field $J$ of type $(1,1)$ by

$$
J\left(X, f \frac{\mathrm{d}}{\mathrm{d} t}\right)=\left(\phi X-f \xi, \eta(X) \frac{\mathrm{d}}{\mathrm{d} t}\right)
$$

for any $X \in \Gamma(T M)$ and $f \in C^{\infty}(M \times \mathbb{R})$. One easily checks that $J^{2}=-I$, giving an almost complex structure on $M \times \mathbb{R}$. Then one says that, by definition, the almost contact structure $(\phi, \xi, \eta)$ is normal if $J$ is a complex structure. Some long but straightforward computations of the Nijenhuis torsion of $J$ yield, for any $X, Y \in \Gamma(T M)$,

$$
\begin{aligned}
& {[J, J]((X, 0),(Y, 0))=\left(N^{(1)}(X, Y), N^{(2)}(X, Y) \frac{\mathrm{d}}{\mathrm{d} t}\right)} \\
& {[J, J]\left((X, 0),\left(0, \frac{\mathrm{d}}{\mathrm{d} t}\right)\right)=\left(N^{(3)}(X), N^{(4)}(X) \frac{\mathrm{d}}{\mathrm{d} t}\right),}
\end{aligned}
$$

where we have put

$$
\begin{gathered}
N^{(1)}:=[\phi, \phi]+2 \mathrm{~d} \eta \otimes \xi \\
N^{(2)}(X, Y):=\left(\mathcal{L}_{\phi X} \eta\right)(Y)-\left(\mathcal{L}_{\phi Y} \eta\right)(X) \\
N^{(3)}:=\mathcal{L}_{\xi} \phi \\
N^{(4)}:=\mathcal{L}_{\xi} \eta .
\end{gathered}
$$

Therefore $(\phi, \xi, \eta)$ is normal if and only if all the above fundamental tensors $N^{(1)}, N^{(2)}, N^{(3)}, N^{(4)}$ vanish identically. However, it was proved that the vanishing of $N^{(1)}$ implies the vanishing of the remaining tensors. Thus one has the following theorem.

Theorem 3.1 ([135]). An almost contact manifold $(M, \phi, \xi, \eta)$ is normal if and only if the tensor field $N^{(1)}$ vanishes identically.

A Riemannian metric $g$ on $M$ is said to be compatible with the almost contact structure if it satisfies

$$
g(\phi X, \phi Y)=g(X, Y)-\eta(X) \eta(Y)
$$

for any $X, Y \in \Gamma(T M)$. Such a metric always exists, provided that $M$ is paracompact. If we fix one, say $g$, we call the geometric structure $(\phi, \xi, \eta, g)$ an almost contact metric structure. Note that, taking $Y=\xi$ in (3.6) we get

$$
\eta(X)=g(X, \xi) .
$$

Moreover, (3.6) implies that $g(\phi X, Y)=-g(X, \phi Y)$ for any $X, Y \in \Gamma(T M)$, so that the bilinear form $\Phi$ defined by

$$
\Phi(X, Y):=g(X, \phi Y)
$$

is in fact a 2-form, called fundamental 2-form (or Sasaki form) of the almost contact metric manifold $(M, \phi, \xi, \eta, g)$. Since $\phi \xi=0$ one easily gets

$$
i_{\xi} \Phi=0
$$

Due to (3.7), we can decompose the tangent bundle of an almost contact metric manifold as the orthogonal sum of the codimension 1 distribution $\mathcal{D}=\operatorname{ker}(\eta)$ and the 1-dimensional distribution $\mathcal{V}$ defined by the vector field $\xi$.

There are many remarkable classes of almost contact metric manifolds (for a full classification see [43]). One of them is given by quasi-Sasakian manifolds, that is normal almost contact metric manifolds whose fundamental 2-form is closed. This class of almost contact metric manifolds, which resembles the class of Kähler manifolds in even dimension, was defined by Blair in his Ph.D. thesis (see 12]) and then studied 
by several authors. Among Blair's results there is the fact that there are no quasi-Sasakian manifolds of even rank. Recall that an almost contact manifold $(M, \phi, \xi, \eta)$ of dimension $2 n+1$ is said to be of rank $2 p+1$ if, for some $p \leq n$, one has that $\eta \wedge(\mathrm{d} \eta)^{p} \neq 0$ at any point of $M$ and $(\mathrm{d} \eta)^{p+1} \equiv 0$, whereas one says that $M$ has rank $2 p$ if $(\mathrm{d} \eta)^{p} \neq 0$ and $\eta \wedge(\mathrm{d} \eta)^{p} \equiv 0$ on $M$. If the rank is maximal, $\eta$ becomes a contact form. We now examine the minimal rank case.

Definition 3.2. An almost coKähler manifold is an almost contact metric manifold $(M, \phi, \xi, \eta, g)$ such that the fundamental 2-form $\Phi$ and the 1-form $\eta$ are closed. If in addition the almost contact structure is normal, we say that $M$ is a coKähler manifold.

Any almost coKähler manifold is canonically a cosymplectic manifold, with cosymplectic structure determined by the 1 -form $\eta$, the 2 -form $\Phi$, and $\xi$ as Reeb vector field. This can be proved by considering a $\phi$-basis, that is a local orthonormal basis $\left\{X_{1}, \ldots, X_{n}, Y_{1}, \ldots, Y_{n}, \xi\right\}$, where for each $i \in\{1, \ldots, n\}$, $Y_{i}=\phi X_{i}$. Such a basis exists in every almost contact metric manifold (see e.g. page 44 of [15]). Then

$$
\eta \wedge \Phi^{n}\left(\xi, X_{1}, \ldots, X_{n}, Y_{1}, \ldots, Y_{n}\right) \neq 0 .
$$

Conversely, any cosymplectic manifold carries an associated almost coKähler structure, as shown in the following theorem.

Theorem 3.3 (cf. [14, 58]). Let $(M, \eta, \omega)$ be an almost cosymplectic manifold of dimension $2 n+1$ with Reeb vector field $\xi$. Then, there exists an almost contact metric structure $(\phi, \xi, \eta, g)$ on $M$ (with the same $\eta$ and $\xi)$, whose fundamental 2 -form $\Phi$ coincides with $\omega$.

Proof. Let $\xi$ be the Reeb vector field of $(M, \eta, \omega)$ (see page 4). It satisfies the equalities

$$
\eta(\xi)=1, \quad i_{\xi} \omega=0 .
$$

For every non-zero $X \in \Gamma(\operatorname{ker}(\eta))$, we have $i_{X} \omega=i_{X} \omega+\eta(X) \eta=b_{(\eta, \omega)}(X)$, which is non-zero since $b_{(\eta, \omega)}$ is an isomorphism. This shows that the restriction of $\omega$ to $\mathcal{D}:=\operatorname{ker}(\eta)$ is a non-degenerate quadratic form. Denote by $\mathbb{R} \xi$ the distribution generated by $\xi$. Then there exists a vector bundle endomorphism $\phi_{\mathcal{D}}: \mathcal{D} \rightarrow \mathcal{D}$ and a metric $g_{\mathcal{D}}$ on $\mathcal{D}$ such that for any vector fields $X, Y \in \Gamma(\mathcal{D})$,

$$
g_{\mathcal{D}}\left(X, \phi_{\mathcal{D}} Y\right)=\omega(X, Y),
$$

and $\phi_{\mathcal{D}}^{2}=-I_{\mathcal{D}}$, where $I_{\mathcal{D}}$ denotes the identity map on $\mathcal{D}$ (see for example [95]). Now, we define a metric $g$ on $M$ by

$$
g(X, Y)=g_{\mathcal{D}}(X, Y), \quad g(X, \xi)=0, \quad g(\xi, \xi)=1,
$$

for any $X, Y \in \Gamma(\mathcal{D})$. Next, we define a vector bundle endomorphism $\phi: T M \rightarrow T M$ by

$$
\phi(X)=\phi_{\mathcal{D}}(X), \quad \phi(\xi)=0
$$

for any $X \in \Gamma(\mathcal{D})$. Then, it is easy to check that $(M, \phi, \xi, \eta, g)$ is an almost contact metric structure and

$$
g(X, \phi Y)=\omega(X, Y) .
$$

Corollary 3.4. Any cosymplectic manifold $(M, \eta, \omega)$ admits an almost coKähler structure $(\phi, \xi, \eta, g)$ with fundamental 2-form $\Phi=\omega$.

CoKähler manifolds were studied for the first time in [12] and [16] in the context of quasi-Sasakian manifolds, since they are quasi-Sasakian manifolds of rank 1.

We collect some basic properties of almost coKähler manifolds. The first is that, due to the closedness of $\eta$, one has that $\mathcal{D}$ is integrable and, moreover, the Reeb vector field is an infinitesimal automorphism with respect to $\mathcal{D}$, that is $[\xi, X] \in \Gamma(\mathcal{D})$ for any $X \in \Gamma(\mathcal{D})$.

Notice that, by (3.8) and the Cartan formula for the Lie derivative, one obtains

$$
\mathcal{L}_{\xi} \Phi=0 .
$$


The next theorem shows some properties which almost coKähler manifolds share with contact metric manifolds (cf. [15, Theorem 6.2]):

Theorem 3.5. For an almost coKähler structure $(\phi, \xi, \eta, g)$ the tensor fields $N^{(2)}$ and $N^{(4)}$ vanish. Moreover, $N^{(1)}$ coincides with the Nijenhuis tensor of $\phi$ and $N^{(3)}$ vanishes if and only if $\xi$ is a Killing vector field.

Proof. The claim straightforwardly follows once one observes that $N^{(2)}$ and $N^{(4)}$ can be written as

$$
N^{(2)}(X, Y)=2 \mathrm{~d} \eta(\phi X, Y)-2 \mathrm{~d} \eta(X, \phi Y), \quad N^{(4)}(X)=2 \mathrm{~d} \eta(\xi, X) .
$$

For the last part of the theorem, notice that by (3.9) one has

$$
0=\left(\mathcal{L}_{\xi} \Phi\right)(X, Y)=\left(\mathcal{L}_{\xi} g\right)(X, \phi Y)+g\left(X,\left(\mathcal{L}_{\xi} \phi\right) Y\right),
$$

from which, by using the vanishing of $N^{(4)}$, the assertion follows.

Corollary 3.6. In any almost coKähler manifold the integral curves of the Reeb vector field are geodesics.

Proof. The vanishing of $N^{(4)}$ yields, for any $X \in \Gamma(T M)$,

$$
\begin{aligned}
0 & =\xi(\eta(X))-\eta([\xi, X]) \\
& =\xi(g(X, \xi))-g\left(\nabla_{\xi} X, \xi\right)+g\left(\nabla_{X} \xi, \xi\right) \\
& =g\left(X, \nabla_{\xi} \xi\right),
\end{aligned}
$$

since $g\left(\nabla_{X} \xi, \xi\right)=X(g(\xi, \xi))-g\left(\xi, \nabla_{X} \xi\right)$, from which it follows that $2 g\left(\nabla_{X} \xi, \xi\right)=0$.

Another general property of almost coKähler manifolds concerns the harmonicity of the forms $\eta$ and $\Phi:$

Theorem $3.7(\sqrt[90]{)})$. The 1-form $\eta$ and the 2-form $\Phi$ of any almost coKähler manifold are harmonic.

Now recall the following general formula, which holds for any almost contact metric manifold ( 15 . Lemma 6.1]),

$$
\begin{aligned}
2 g\left(\left(\nabla_{X} \phi\right) Y, Z\right)= & 3 \mathrm{~d} \Phi(X, \phi Y, \phi Z)-3 \mathrm{~d} \Phi(X, Y, Z)+g\left(N^{(1)}(Y, Z), \phi X\right) \\
& +N^{(2)}(Y, Z) \eta(X)+2 \mathrm{~d} \eta(\phi Y, X) \eta(Z)-2 \mathrm{~d} \eta(\phi Z, X) \eta(Y) .
\end{aligned}
$$

Therefore we have the following result for almost coKähler manifolds.

Corollary 3.8. In any almost coKähler manifold one has

$$
g\left(\left(\nabla_{X} \phi\right) Y, Z\right)=\frac{1}{2} g([\phi, \phi](Y, Z), \phi X) .
$$

From (3.10) it follows in particular that

$$
\nabla_{\xi} \phi=0
$$

which was already proved in another way by Olszak ([125). Another easy consequence of (3.10) is that if $M$ is coKähler then $\phi$ is parallel. However also the converse holds. In fact we have the following more general result.

Theorem 3.9 (12]). An almost contact metric manifold $(M, \phi, \xi, \eta, g)$ is coKähler if and only if $\nabla \phi=0$ or, equivalently, $\nabla \Phi=0$.

On an almost coKähler manifold it is often convenient to use the operator

$$
h:=\frac{1}{2} \mathcal{L}_{\xi} \phi=\frac{1}{2} N^{(3)} .
$$

The main properties of this tensor field are described in the following proposition, which can be proved straightforwardly (see also [67]). 
Proposition 3.10. Let $(M, \phi, \xi, \eta, g)$ be an almost coKähler manifold. Then the operator $h$ satisfies the following properties:

(i) $h$ is a symmetric operator with respect to $g$;

(ii) $h$ anti-commutes with $\phi$;

(iii) $\nabla \xi=-\phi h$.

Then we can state the following important result.

Theorem 3.11. Let $(M, \phi, \xi, \eta, g)$ be an almost coKähler manifold. The following statements are equivalent:

(a) the operator $h$ vanishes identically;

(b) the Reeb vector field is parallel;

(c) the Reeb vector field is Killing;

(d) the foliation $\mathcal{D}$ defined by the kernel of $\eta$ is totally geodesic;

(e) $M$ is locally the Riemannian product of an almost Kähler manifold with the real line;

(f) $\nabla \eta=0$.

Remark 3.12. Since in any coKähler manifold, as $N^{(1)}=0$, also the tensor field $N^{(3)}$ vanishes, by Theorem 3.11 we have that $\xi$ is Killing. The converse, in general, does not hold. However, it was proved that in dimension 3 any among the above six conditions is equivalent to requiring that the manifold is coKähler (90]).

Concerning the operator $h$, we state the following general identity, due to Olszak ([125]), which relates $h$ with the covariant derivative of $\phi$ in any almost coKähler manifold

$$
\left(\nabla_{\phi X} \phi\right) \phi Y+\left(\nabla_{X} \phi\right) Y-\eta(Y) h X=0 .
$$

Notice that, from (3.12) it follows that $\left(\nabla_{\phi X} \eta\right)(\phi Y)=-\left(\nabla_{X} \eta\right)(Y)$.

3.2. Examples of coKähler manifolds. The standard example of (almost) coKähler manifold is given by the product of an (almost) Kähler manifold $(N, J, G)$ with $\mathbb{R}\left(\right.$ or $\left.S^{1}\right)$. If $t$ denotes the global coordinate on $\mathbb{R}$, any vector field of $M$ can be written as $\left(X, f \frac{\mathrm{d}}{\mathrm{d} t}\right)$ for some smooth function $f$ on the product. Let us define a tensor field $\phi$ of type $(1,1)$ by setting $\phi\left(X, f \frac{\mathrm{d}}{\mathrm{d} t}\right):=(J X, 0)$, a vector field $\xi:=\frac{\mathrm{d}}{\mathrm{d} t}$, a 1 -form $\eta:=\mathrm{d} t$ and a Riemannian metric $g$ as the product metric $G+\mathrm{d} t^{2}$. Then one can check that $(\phi, \xi, \eta, g)$ is an almost coKähler structure such that $\xi$ is Killing, and it is coKähler if and only if $N$ is Kähler.

On the other hand by Theorem 3.11 every coKähler manifold is, at least locally, the Riemannian product of a Kähler manifold with the real line.

Therefore two natural problems rise:

Question 1 - Are there examples of almost coKähler manifolds which are not, even locally, the Riemannian product of an almost Kähler manifold with the real line?

Question 2 - Are there examples of coKähler manifolds which are not the global product of a Kähler manifold with $\mathbb{R}$ or $S^{1}$ ?

Clearly, in view of Theorem 3.11. Question 1 is equivalent to the problem of finding examples of proper almost coKähler manifolds with non-Killing Reeb vector field. The problem was solved by Olszak (125), who found an example of an almost coKähler structure with non-parallel Reeb vector field in any odd dimension. Namely, let $\mathfrak{g}$ be the $(2 n+1)$-dimensional real solvable Lie algebra with basis $\left\{E_{0}, E_{1}, \ldots, E_{2 n}\right\}$ and non-zero Lie brackets

$$
\left[E_{0}, E_{i}\right]=-a_{i} E_{i}-a_{i+n} E_{i+n}, \quad\left[E_{0}, E_{i+n}\right]=-a_{i+n} E_{i}+a_{i} E_{i+n},
$$

where $a_{1}, \ldots, a_{2 n}$ are real numbers such that $a_{1}^{2}+\cdots+a_{2 n}^{2}>0$. Let $G$ be a Lie group whose Lie algebra is $\mathfrak{g}$ and extend $\left\{E_{0}, E_{1}, \ldots, E_{2 n}\right\}$ to left invariant vector fields on $G$. We define on $G$ a Riemannian 
metric $g$ by imposing that $\left\{E_{0}, E_{1}, \ldots, E_{2 n}\right\}$ is orthonormal. Then we define a 1-form $\eta$ and a tensor field $\phi$ by setting $\eta\left(E_{j}\right)=\delta_{0 j}$ for each $j \in\{0,1, \ldots, 2 n\}$ and $\phi E_{0}=0, \phi E_{i}=E_{i+n}, \phi E_{i+n}=-E_{i}$ for each $i \in\{1, \ldots, n\}$. Then putting $\xi:=E_{0}$, one can prove that $(\phi, \xi, \eta, g)$ is an almost coKähler structure such that $\nabla \xi \neq 0$.

With regard to Question 2, it was positively solved in dimension 3 by Chinea, de León, Marrero in 1993 (40]). We now describe such an example explicitly. Firstly let us consider the following general construction, due to Fujimoto and $\operatorname{Mutō}([83)$. Let $(N, J, G)$ be a compact Kähler manifold and $f$ : $N \longrightarrow N$ be a Hermitian isometry, that is an isometry of the Riemannian manifold $(N, g)$ such that $f_{*} \circ J=J \circ f_{*}$. Define an action $\rho: \mathbb{Z} \times(N \times \mathbb{R}) \longrightarrow N \times \mathbb{R}$ of $\mathbb{Z}$ on $N \times \mathbb{R}$ by $\rho(k,(x, t))=\left(f^{k}(x), t+k\right)$. This action is free and properly discontinuous, so that the corresponding orbit space $M_{f}$ is a compact smooth manifold. Then the coKähler structure induced on the product manifold $N \times \mathbb{R}$ descends to a coKähler structure on $M_{f}:=(N \times \mathbb{R}) / \mathbb{Z}$. Now Chinea, de León, Marrero restrict to the case when $N$ is the 2-dimensional torus $\mathbb{T}^{2}$ with its standard Kähler structure and $f$ is the Hermitian isometry induced by the isometry of $\mathbb{R}^{2} f(x, y)=(y,-x)$. They note that the manifold $M_{f}:=\left(\mathbb{T}^{2} \times \mathbb{R}\right) / \mathbb{Z}$ is nothing but the manifold $\mathbb{T}_{A}^{3}$ considered in [86], where

$$
A=\left(\begin{array}{cc}
0 & 1 \\
-1 & 0
\end{array}\right)
$$

As, according to [86], $A$ is neither conjugated with a matrix of the form

$$
A=\left(\begin{array}{ll}
1 & n \\
0 & 1
\end{array}\right)
$$

nor

$$
A=\left(\begin{array}{cc}
-1 & n \\
0 & 1
\end{array}\right)
$$

the first Betti number of $M_{f}$ is 1 . Therefore, $M_{f}$ cannot be the global product of a compact surface $S$ with the unit circle $S^{1}$. Indeed in such a case the first Betti number of $S$ would be 0 and hence $S$ would be the sphere $S^{2}$. Then from the homology exact sequence

$$
0 \longrightarrow \mathbb{Z}^{2} \longrightarrow \Pi_{1}\left(\mathbb{T}_{A}^{3}\right) \longrightarrow \mathbb{Z} \longrightarrow 0
$$

one would get an injective homomorphism from $\mathbb{Z}^{2}$ into $\Pi_{1}\left(\mathbb{T}_{A}^{3}\right)=\mathbb{Z}$, which yields a contradiction. This is the argument used in 40 for proving that $M_{f}$ is not the global product of a Kähler manifold with $S^{1}$.

In 115, Marrero and Padrón generalized this example to any odd dimension. We briefly illustrate their construction. Let us consider the $2 n$-dimensional torus $\mathbb{T}^{2 n}$ with the Kähler structures $(J, G)$ and $\left(J^{\prime}, G^{\prime}\right)$ defined as follows

$$
\begin{gathered}
J X_{i}=-Y_{i}, \quad J Y_{i}=X_{i}, \quad G=\sum_{j=1}^{2 n}\left(\alpha_{j} \otimes \alpha_{j}+\beta_{j} \otimes \beta_{j}\right) \\
J^{\prime} X_{i}^{\prime}=-Y_{i}^{\prime}, \quad J^{\prime} Y_{i}^{\prime}=X_{i}^{\prime}, \quad G^{\prime}=\sum_{j=1}^{2 n}\left(\alpha_{j}^{\prime} \otimes \alpha_{j}^{\prime}+\beta_{j}^{\prime} \otimes \beta_{j}^{\prime}\right)
\end{gathered}
$$

for each $i \in\{1, \ldots, n\}$, where $\left\{X_{1}, \ldots, X_{n}, Y_{1}, \ldots, Y_{n}\right\}$ is the canonical global basis of vector fields on $\mathbb{T}^{2 n}$, $\left\{\alpha_{1}, \ldots, \alpha_{n}, \beta_{1}, \ldots, \beta_{n}\right\}$ the corresponding dual basis of 1 -forms, $\alpha_{i}^{\prime}:=\alpha_{i}+\cos \frac{\pi}{3} \beta_{i}, \beta_{i}^{\prime}:=-\sin \frac{\pi}{3} \beta_{i}$ and $\left\{X_{1}^{\prime}, \ldots, X_{n}^{\prime}, Y_{1}^{\prime}, \ldots, Y_{n}^{\prime}\right\}$ the dual basis of vector fields of the basis of 1 -forms $\left\{\alpha_{1}^{\prime}, \ldots, \alpha_{n}^{\prime}, \beta_{1}^{\prime}, \ldots, \beta_{n}^{\prime}\right\}$.

Then, as described before, we can consider the action of $\mathbb{Z}$ on $\mathbb{T}^{2 n} \times \mathbb{R}$ via an Hermitian isometry $f$ and the corresponding quotient space $M_{f}:=\left(\mathbb{T}^{2 n} \times \mathbb{R}\right) / \mathbb{Z}$. Let us consider the case when $f$ is induced 
by an isometry $\tilde{f}$ of $\mathbb{R}^{2 n}$. Marrero and Padrón considered the following four cases

$$
\begin{gathered}
\tilde{f}_{1}\left(x_{1}, \ldots, x_{n}, y_{1}, \ldots, y_{n}\right)=\left(y_{1}, \ldots, y_{n},-x_{1}, \ldots,-x_{n}\right) \\
\tilde{f}_{2}\left(x_{1}, \ldots, x_{n}, y_{1}, \ldots, y_{n}\right)=\left(-x_{1}, \ldots,-x_{n},-y_{1}, \ldots,-y_{n}\right) \\
\tilde{f}_{3}\left(x_{1}, \ldots, x_{n}, y_{1}, \ldots, y_{n}\right)=\left(-y_{1}, \ldots,-y_{n}, x_{1}+y_{1}, \ldots, x_{n}+y_{n}\right) \\
\tilde{f}_{4}\left(x_{1}, \ldots, x_{n}, y_{1}, \ldots, y_{n}\right)=\left(-x_{1}-y_{1}, \ldots,-x_{n}-y_{n}, x_{1}, \ldots, x_{n}\right),
\end{gathered}
$$

so obtaining the corresponding compact coKähler manifolds $M_{f_{i}}, i \in\{1, \ldots, 4\}$. Notice that, for $n=1$, $M_{f_{1}}$ coincides with the 3-dimensional example of Chinea, de León, Marrero illustrated before. Then the authors proved the following remarkable result:

Theorem 3.13 ([115). The manifolds $M_{f_{1}}, M_{f_{2}}, M_{f_{3}}, M_{f_{4}}$ are $(2 n+1)$-dimensional compact flat coKähler solvmanifolds which are not topologically equivalent to the compact coKähler manifolds $\mathbb{T}^{2 m+1} \times$ $\mathbb{C} P^{r}$, with $m, r>0, m+r=n$.

Concerning the above examples, Fino and Vezzoni (81]) showed that these solvmanifolds are finite quotients of a torus. In fact the authors prove a more general result, namely that a solvmanifold has a coKähler structure if and only if it is a finite quotient of a torus which has a structure of a torus bundle over a complex torus. Recently, in [9] Bazzoni and Oprea found non-trivial examples of non-product compact coKähler manifolds in every odd dimension.

3.3. CoKähler manifolds and Kähler geometry. CoKähler manifolds are the most natural analogues in odd dimension of Kähler manifolds. This can be seen for instance by looking at the 1-dimensional foliation $\mathcal{V}$ defined by the Reeb vector field, usually called Reeb foliation. In fact notice that the closedness of the fundamental 2-form $\Phi$ together with (3.8) implies that $\Phi$ is a basic 2-form. Moreover, by Theorem 3.11 the vanishing of $N_{\phi}^{(3)}$ also implies that the tensor field $\phi$ and the Riemannian metric $g$ are projectable tensors. Roughly speaking, this means that $\Phi, \phi$ and $g$ are constant along the leaves of the Reeb foliation, so that they locally project to a 2 -form $\omega^{\prime}$, a tensor field $J^{\prime}$ and a Riemannian metric $G^{\prime}$ on the leaf space. Then $\left(\omega^{\prime}, J^{\prime}, G^{\prime}\right)$ form a Kähler structure. Thus any coKähler manifold is foliated by a transversely Kähler foliation. If in particular $\mathcal{V}$ is simple, then there exists a Riemannian submersion $\pi:(M, g) \longrightarrow\left(N^{\prime}, G^{\prime}\right)$ such that $\operatorname{ker}\left(\pi_{*}\right)=T \mathcal{V}$ and $\pi_{*} \circ \phi=J^{\prime} \circ \pi_{*}$.

Therefore, at least locally, any coKähler manifold projects onto a Kähler one. On the other hand, given a coKähler manifold $M$ there exists a Kähler manifold $N$ projecting onto $M$. It is enough to consider the product of $M$ with the real line (or the unit circle), endowed with the complex structure $J$ defined by (3.1) and the product metric $G=g+\mathrm{d} t^{2}$. An easy computation shows that $G$ is an Hermitian metric. Moreover, one has

$$
\begin{aligned}
\omega\left(\left(X, f \frac{\mathrm{d}}{\mathrm{d} t}\right),\left(Y, h \frac{\mathrm{d}}{\mathrm{d} t}\right)\right) & =G\left(\left(X, f \frac{\mathrm{d}}{\mathrm{d} t}\right), J\left(Y, h \frac{\mathrm{d}}{\mathrm{d} t}\right)\right) \\
& =\Phi(X, Y)-h \eta(X)+f \eta(Y),
\end{aligned}
$$

from which it follows that

$$
\mathrm{d} \omega\left(\left(X, f \frac{\mathrm{d}}{\mathrm{d} t}\right),\left(Y, h \frac{\mathrm{d}}{\mathrm{d} t}\right),\left(Z, k \frac{\mathrm{d}}{\mathrm{d} t}\right)\right)=\mathrm{d} \Phi(X, Y, Z)-\frac{2}{3}(k \mathrm{~d} \eta(X, Y)-h \mathrm{~d} \eta(X, Z)+f \mathrm{~d} \eta(Y, Z)),
$$

for any $X, Y, Z \in \Gamma(T M)$ and $f, h, k \in C^{\infty}(M)$. Therefore, the almost Hermitian structure induced on $M \times \mathbb{R}$ is (almost) Kähler if and only if $M$ is (almost) coKähler.

This construction on the product of an almost contact manifold with the real line can be extended to a more general setting. Let $\left(M_{1}, \phi_{1}, \xi_{1}, \eta_{1}\right)$ and $\left(M_{2}, \phi_{2}, \xi_{2}, \eta_{2}\right)$ be almost contact manifolds. We define on the product manifold $M_{1} \times M_{2}$ a tensor field $J$ by setting

$$
J\left(X_{1}, X_{2}\right)=\left(\phi_{1} X_{1}-\eta_{2}\left(X_{2}\right) \xi_{1}, \phi_{2} X_{2}+\eta_{1}\left(X_{1}\right) \xi_{2}\right) .
$$


One checks that $J^{2}=-I$, so that an almost complex structure is defined on $M_{1} \times M_{2}$. This construction is due to Morimoto ([121]), who proved that $\left(M_{1} \times M_{2}, J\right)$ is a complex manifold if and only if $\left(M_{1}, \phi_{1}, \xi_{1}, \eta_{1}\right)$ and $\left(M_{2}, \phi_{2}, \xi_{2}, \eta_{2}\right)$ are normal almost contact manifolds. It is worth mentioning that an interesting corollary of this is the well-known result, previously proved by Calabi and Eckmann, that the product $S^{2 p+1} \times S^{2 q+1}$ of any two odd-dimensional spheres is a complex manifold. Notice that, since the second Betti numbers of these compact manifolds are zero, they cannot carry any Kähler structure.

Now let us consider two almost contact metric manifolds $\left(M_{1}, \phi_{1}, \xi_{1}, \eta_{1}, g_{1}\right)$ and $\left(M_{2}, \phi_{2}, \xi_{2}, \eta_{2}, g_{2}\right)$. Then Goldberg ([88]) proved that the product $M_{1} \times M_{2}$, endowed with the above almost complex structure $J$ and with the product metric $G=g_{1}+g_{2}$ is Kähler, provided that the factors are coKähler. Later on, Capursi (35) proved that also the converse holds. In fact he proved the result in a more general setting, dealing with almost Kähler and nearly Kähler manifolds. Summing up, we can state the following theorem.

Theorem 3.14. The almost Hermitian manifold $\left(M_{1} \times M_{2}, J, G\right)$ is (almost) Kähler if and only if both $\left(M_{1}, \phi_{1}, \xi_{1}, \eta_{1}, g_{1}\right)$ and $\left(M_{2}, \phi_{2}, \xi_{2}, \eta_{2}, g_{2}\right)$ are (almost) coKähler.

In [150] Watson further generalized Morimoto's construction by considering, for each real number $a$ and $b \neq 0$ the manifold $M=M_{1} \times M_{2}$ endowed with the tensor field $J_{a, b}$ and the Riemannian metric $G_{a, b}$ defined by

$$
J_{a, b}\left(X_{1}, X_{2}\right)=\left(\phi_{1} X_{1}-\left(\frac{a}{b} \eta_{1}\left(X_{1}\right)+\frac{a^{2}+b^{2}}{b} \eta_{2}\left(X_{2}\right)\right) \xi_{1}, \phi_{2} X_{2}+\left(\frac{1}{b} \eta_{1}\left(X_{1}\right)+\frac{a}{b} \eta_{2}\left(X_{2}\right)\right) \xi_{2}\right)
$$

and

$$
\begin{aligned}
G_{a, b}\left(\left(X_{1}, X_{2}\right),\left(Y_{1}, Y_{2}\right)\right)= & g_{1}\left(X_{1}, Y_{1}\right)+a \eta_{1}\left(X_{1}\right) \eta_{2}\left(Y_{2}\right)+a \eta_{1}\left(Y_{1}\right) \eta_{2}\left(X_{2}\right) \\
& +\left(a^{2}+b^{2}+1\right) \eta_{2}\left(X_{2}\right) \eta_{2}\left(Y_{2}\right)+g_{2}\left(X_{2}, Y_{2}\right) .
\end{aligned}
$$

In the most general case, that is when $\left(M_{1}, \phi_{1}, \xi_{1}, \eta_{1}, g_{1}\right)$ and $\left(M_{2}, \phi_{2}, \xi_{2}, \eta_{2}, g_{2}\right)$ are almost contact metric manifolds, one has that $\left(M, J_{a, b}, G_{a, b}\right)$ is an almost Hermitian manifold. The fundamental 2 -form $\omega_{a, b}:=$ $G\left(\cdot, J_{a, b} \cdot\right)$ is then given by

$$
\omega_{a, b}=\Phi_{1}+\Phi_{2}+2 b \eta_{1} \wedge \eta_{2}
$$

from which

$$
\mathrm{d} \omega_{a, b}=\mathrm{d} \Phi_{1}+\mathrm{d} \Phi_{2}+2 b\left(\mathrm{~d} \eta_{1} \wedge \eta_{2}-\eta_{1} \wedge \mathrm{d} \eta_{2}\right) .
$$

Again, one proves that $\left(M, J_{a, b}, G_{a, b}\right)$ is a Kähler manifold if and only each $\left(M_{i}, \phi_{i}, \xi_{i}, \eta_{i}, g_{i}\right)$ is coKähler, $i \in\{1,2\}$. Then Watson used the aforementioned Olszak's examples of strictly almost coKähler non-compact solvable Lie groups for constructing new examples of almost Kähler non-Kähler manifolds. It is enough to take the product of any of such Lie groups and to apply the previous results on the almost Kähler structure on the products. Namely, he proved the following result.

Theorem 3.15. Given two odd integers, $2 p+1$ and $2 q+1, p, q>0$, there exist noncompact, connected, solvable Lie groups $G_{1}^{2 p+1}$ and $G_{2}^{2 q+1}$, such that $G=G_{1}^{2 p+1} \times G_{2}^{2 q+1}$ carries a two parameter family of almost Kähler structures which are not Kähler.

Further, one can consider Thurston's torus bundle $W^{4}$ over $\mathbb{T}^{2}$ with strictly almost Kähler structure $(J, g)$. Then one puts on $W^{4} \times S^{1}$ the standard almost coKähler structure, so that $W^{4} \times S^{1}$ cannot be coKähler because $W^{4}$ is not Kähler. Thus, the non-Kähler almost Kähler structure described before is defined on $M=\left(W^{4} \times S^{1}\right) \times\left(W^{4} \times S^{1}\right)$. Note that, as shown in [150], we could not have obtained the non-Kähler property of $M$ from its cohomology. Moreover, if $N$ is any compact Kähler manifold, then $M=\left(W^{4} \times S^{1}\right) \times\left(N \times S^{1}\right)$ can be made strictly almost Kähler in the same way. However, in this case, $b_{1}(M)=b_{1}(N)+5$ is odd and the non-Kähler property emerges from cohomological considerations. 
Turning to the general case, we mentioned that the $2 n$-dimensional distribution $\mathcal{D}:=\operatorname{ker}(\eta)$ of any almost coKähler manifold $M$ of dimension $2 n+1$ is integrable and so defines a foliation orthogonal to the Reeb foliation. Since $\phi X \in \Gamma(\mathcal{D})$ for any $X \in \Gamma(\mathcal{D})$, $\phi$ induces an almost complex structure $J$ on each leaf of $\mathcal{D}$, which is compatible with the metric $G$ induced by $g$. One can easily see that the fundamental 2 -form of this almost Hermitian structure is closed, so that we have that the leaves of $\mathcal{D}$ are almost Kähler manifolds. Olszak proposed the following definition ([127). An almost coKähler manifold with Kählerian leaves is an almost coKähler manifold such that the leaves of the canonical foliation $\mathcal{D}$ are Kähler manifolds. Clearly if $M$ is coKähler or if $\operatorname{dim}(M)=3$ then $M$ is an almost coKähler manifold with Kählerian leaves. Thus the question is whether in dimension greater than 3 any almost coKähler manifold with Kählerian leaves is necessarily coKähler. Olszak gave a negative answer, by finding some explicit counterexamples (see Example 3 and 4 in [127). Moreover he proved the following characterization.

Theorem 3.16 ([127). Let $(M, \phi, \xi, \eta, g)$ be an almost coKähler manifold. Then $M$ is almost coKähler with Kählerian leaves if and only if the structure $(\phi, \xi, \eta, g)$ fulfils the condition

$$
\left(\nabla_{X} \phi\right) Y=g(X, h Y) \xi-\eta(Y) h X
$$

for any $X, Y \in \Gamma(T M)$.

Corollary 3.17. An almost coKähler manifold with Kählerian leaves is coKähler if and only if the Reeb vector field is Killing.

We remarked that in dimension 3 any almost coKähler manifold with $\xi$ Killing is necessarily coKähler, but in higher dimensions this is not true. Thus in view of Corollary 3.17, we can say that the condition of having Kählerian leaves is what is missing for an almost coKähler manifold with $\xi$ Killing to be coKähler.

Furthermore, we mention that recently Dacko has developed a new approach towards almost coKähler manifolds with Kählerian leaves based on the techniques of CR-geometry (see [49] for more details).

3.4. CoKähler structures on hypersurfaces of Kähler manifolds. Other examples come from hypersurfaces of Kähler manifolds. The first author that studied the geometric structures induced on a hypersurface of an almost Hermitian manifold was Tashiro in 1963 ([141], 142]). A few years later Okumura ([124]) and Goldberg [88 found necessary and sufficient conditions for which a hypersurface of a Kähler manifold carries a coKähler structure. Let $(\tilde{M}, \tilde{J}, \tilde{g})$ be a $(2 n+2)$-dimensional Kähler manifold and let $\iota: M \longrightarrow \tilde{M}$ be a $C^{\infty}$ orientable hypersurface. Let $N$ be the unit normal of $M$ with orientation determined by that of $M$. Then for any vector field $X \in \Gamma(T M)$ let us decompose $\tilde{J}_{\iota_{*}} X$ into the components tangent and normal to $M$, so defining a (1,1)-tensor field $\phi$ and 1-form $\eta$ on $M$ by

$$
\tilde{J}_{\iota_{*}} X=\iota_{*} \phi X+\eta(X) N .
$$

Next, one can prove that $\xi:=-\tilde{J} N$ is a vector field tangent to $M$ and that $(\phi, \xi, \eta, g)$ is an almost contact metric structure, where $g:=\iota^{*} \tilde{g}$ is the induced metric. Now we have the following theorem:

Theorem 3.18 ([124], 88]). With the above notation, a necessary and sufficient condition for a smooth orientable hypersurface $M$ of a Kähler manifold $\tilde{M}$ to be almost coKähler is that its Weingarten operator $A$ anticommutes with $\phi$,

$$
A \phi+\phi A=0 .
$$

A necessary and sufficient condition for $M$ to be coKähler is that its second fundamental form $h$ is proportional to $\eta \otimes \eta$, that is

where $f=h(\xi, \xi)$, or equivalently

$$
h=f \eta \otimes \eta,
$$

$$
A=f \eta \otimes \xi
$$


In particular it follows that any totally geodesic $C^{\infty}$ orientable hypersurface of a Kähler manifold carries a coKähler structure. Further, Okumura studied the geometry of the hypersurfaces in a Kähler manifold of constant holomorphic sectional curvature. He proved that there is no coKähler hypersurface in a Kähler manifold of positive constant holomorphic sectional curvature. This result was improved by Olszak in [126], where the following theorem was proved.

Theorem 3.19 (126]). There are no almost coKähler hypersurfaces in Kähler manifolds of non-zero constant holomorphic sectional curvature.

3.5. Homogeneous coKähler manifolds. An almost contact metric manifold $(M, \phi, \xi, \eta, g)$ is said to be almost contact homogeneous if there exists a connected Lie group $G$ of isometries acting transitively on $M$ and leaving $\phi$ (and hence also $\eta$ and $\xi$, cf. [42]) invariant.

Studying almost contact homogeneous manifolds is a very difficult task in the full generality, so one restricts to some remarkable classes of almost contact metric manifolds. In fact in the coKähler setting there is a full classification in dimension 3, due to a recent work of Perrone ([130]). Namely, a 3dimensional simply connected homogeneous almost coKähler manifold is either a Riemannian product of the real line with a Kähler surface of constant curvature or a Lie group $G$ equipped with a left invariant almost coKähler structure. Moreover, in this last case, we have the following classification, based on the invariant

\section{CoKähler case}

$$
p:=\left\|\mathcal{L}_{\xi} h\right\|-2\|h\|^{2}
$$

(1) If $G$ is unimodular, then the metric is flat and $G$ is either the universal covering $\tilde{E}(2)$ of the group of rigid motions of Euclidean 2-space, or the abelian Lie group $\mathbb{R}^{3}$.

(2) If $G$ is non-unimodular, its Lie algebra $\mathfrak{g}:=\left\langle e_{1}, e_{2}, e_{3}\right\rangle$ is given by

$$
\left[e_{1}, e_{2}\right]=\alpha e_{2}, \quad\left[e_{1}, e_{3}\right]=\left[e_{2}, e_{3}\right]=0, \quad(\alpha \neq 0)
$$

where $e_{1}, e_{2}=\phi e_{1} \in \mathcal{D}$ and $e_{3}=\xi$.

Non-coKähler case

(3) If $G$ is unimodular, then $G$ is one among the following:

- $\tilde{E}(2)$, if $p>0$

- the group $E(1,1)$ of rigid motions of the Minkowski 2-space, if $p<0$

- the Heisenberg group $H^{3}$, if $p=0$.

(4) If $G$ is non-unimodular, then $p=0$ and its Lie algebra $\mathfrak{g}:=\left\langle e_{1}, e_{2}, e_{3}\right\rangle$ is given by

$$
\left[e_{1}, e_{2}\right]=\alpha e_{2}, \quad\left[e_{1}, e_{3}\right]=\gamma e_{2} \quad\left[e_{2}, e_{3}\right]=0, \quad(\alpha, \gamma \neq 0)
$$

where $e_{1}, e_{2}=\phi e_{1} \in \mathcal{D}$ and $e_{3}=\xi$.

Furthermore, Perrone proves that in all the aforementioned cases except (4) the Reeb vector field $\xi$ is harmonic. Recall that a unit vector field $U$ on a $m$-dimensional Riemannian manifold $(M, g)$ can be regarded as a map $U:(M, g) \longrightarrow\left(T_{1} M, g_{S}\right)$, where $g_{S}$ denotes the Sasaki metric on the unit tangent sphere bundle $T_{1} M$. When $M$ is compact we can define the energy of $U$ as the energy of the corresponding map:

$$
E(U)=\frac{1}{2} \int_{M}\|d U\|^{2} \nu_{g}=\frac{m}{2} \operatorname{vol}(M, g)+\frac{1}{2} \int_{M}\|\nabla U\|^{2} \nu_{g} .
$$

A unit vector field $U$ is then called harmonic if it is critical for the energy functional $E$ defined on the set of all unit vector fields on $M$.

A family of examples of homogeneous almost coKähler structures in dimension greater than 3 is proposed in [42. Let us consider the generalized Heisenberg group $H(1, r), r>1$, that is the Lie group 
of real matrices of the form

$$
\left(\begin{array}{cccccc}
1 & 0 & \cdots & 0 & a_{1}^{r+1} & a_{1}^{r+2} \\
0 & 1 & \cdots & 0 & a_{2}^{r+1} & a_{2}^{r+2} \\
\vdots & \vdots & \ddots & \vdots & \vdots & \vdots \\
0 & 0 & \cdots & 1 & a_{r}^{r+1} & a_{r}^{r+2} \\
0 & 0 & \cdots & 0 & 1 & a_{r+1}^{r+2} \\
0 & 0 & \cdots & 0 & 0 & 1
\end{array}\right),
$$

where $a_{i}^{j} \in \mathbb{R}$. This is a connected and simply connected Lie group of dimension $2 r+1$. Let $\left\{x_{i}, y_{i}, z\right\}$, $i \in\{1, \ldots, r\}$, be the coordinate functions on $H(1, r)$ defined by $x_{i}(A)=a_{i}^{r+1}, y_{i}(A)=a_{i}^{r+2}, z(A)=a_{r+1}^{r+2}$, for any $A \in H(1, r)$. One proves that the 1 -forms $\alpha_{i}:=\mathrm{d} x_{i}, \beta_{i}:=\mathrm{d} y_{i}-x_{i} \mathrm{~d} z$ and $\eta:=\mathrm{d} z$ are linearly independent and invariant under the action of $H(1, r)$. Moreover, by setting

$$
\Phi:=-\sum_{i=1}^{r} \alpha_{i} \wedge \beta_{i}
$$

it turns out that $(\eta, \Phi)$ is a cosymplectic structure. A compatible almost coKähler structure is defined in the following way. Let $X_{i}=\frac{\partial}{\partial x_{i}}, Y_{i}:=\frac{\partial}{\partial y_{i}}, \xi:=\frac{\partial}{\partial z}+\sum_{i=1}^{r} x_{i} \frac{\partial}{\partial z}$ be the dual vector fields of the left invariant forms $\alpha_{i}, \beta_{i}, \eta$, respectively. Then one can easily prove that they form an orthonormal frame field with respect to the left invariant metric on $H(1, r)$ defined by

$$
g:=\sum_{i=1}^{r}\left(\alpha_{i}^{2}+\beta_{i}^{2}\right)+\eta \otimes \eta .
$$

Further we define a tensor field $\phi$ by imposing that $g(\cdot, \phi \cdot)=\Phi$. One can check that $(\phi, \xi, \eta, g)$ is an almost coKähler structure. Since $\nabla_{X_{i}} \xi \neq 0$, the structure is not coKähler (cf. [42]). Chinea and Gonzalez give a detailed study of homogeneous structures on $H(1, r)$. In particular, they prove that $H(1, r)$ is a homogeneous almost coKähler manifold of type $\mathcal{T}_{2} \oplus \mathcal{T}_{3}$, according to the classification of homogeneous structures of Tricerri and Vanhecke ([144]).

Further, we also notice that one can consider the manifold $M(1, r)=\Gamma(1, r) \backslash H(1, r)$, where $\Gamma(1, r)$ is the discrete subgroup of $H(1, r)$ of matrices with integer entries. $M(1, r)$ is a compact nilmanifold of dimension $2 r+1$. However, we point out that $M(1, r)$ cannot admit any coKähler structure due to topological obstructions (44). Therefore, as the almost coKähler structure on $H(1, r)$ given above descends to $M(1, r)$, we see that $M(1, r)$ is an example of a compact almost coKähler manifold which cannot have any coKähler structure. Another example of a compact almost coKähler manifold that cannot admit any coKähler structure is $M(1,1) \times \mathbb{T}^{2 r}$ (see 44 for more details).

We notice that some almost contact metric structures on $M(1, r)$ are described in $[39$.

Another class of examples, due to de León ([58), is given by the compact solvmanifolds $M(k)=D_{1} \backslash S_{1}$, where $S_{1}$ is a 3 -dimensional solvable non-nilpotent Lie group and $D_{1}$ a discrete subgroup of $S_{1}$. Namely, let $S_{1}$ be the set of matrices of the form

$$
\left(\begin{array}{cccc}
e^{k z} & 0 & 0 & x \\
0 & e^{-k z} & 0 & y \\
0 & 0 & 1 & z \\
0 & 0 & 0 & 1
\end{array}\right)
$$

where $x, y, z \in \mathbb{R}$ and $k$ is a real number such that $e^{k}+e^{-k}$ is an integer different from 2. One proves that the forms $\mathrm{d} x-k z \mathrm{~d} z, \mathrm{~d} y+k y \mathrm{~d} z$ and $\mathrm{d} z$ constitute a basis of right invariant 1-forms on $S_{1}$. Next, let $D_{1}$ be the discrete subgroup of $S_{1}$ such that the quotient space $M(k)=D_{1} \backslash S_{1}$ is compact (4). Then there exists a global basis $\{\alpha, \beta, \gamma\}$ of 1 -forms on $M(k)$ such that $\pi^{*} \alpha=\mathrm{d} x-k z \mathrm{~d} z, \pi^{*} \beta=\mathrm{d} y+k y \mathrm{~d} z$, 
$\pi^{*} \gamma=\mathrm{d} z$, where $\pi: S_{1} \longrightarrow M(k)$ is the canonical projection. Thus one has $\mathrm{d} \alpha=-k \alpha \wedge \gamma, \mathrm{d} \beta=k \beta \wedge \gamma$ and $\mathrm{d} \gamma=0$. Moreover, if $\{X, Y, Z\}$ is the dual basis of vector fields to $\{\alpha, \beta, \gamma\}$, we have

$$
[X, Y]=0, \quad[X, Z]=k X, \quad[Y, Z]=-k Y .
$$

Now, as observed by de León, $M(k)$ cannot admit any coKähler structure, since otherwise $M(k) \times S^{1}$ would be a Kähler manifold, but it is known that it cannot admit any complex structure (cf. 755). Nevertheless an explicit almost coKähler structure can be defined by putting

$$
\phi:=\alpha \otimes Y-\beta \otimes X, \quad \xi:=Z, \quad \eta:=\gamma, \quad g:=\alpha^{2}+\beta^{2}+\gamma^{2} .
$$

Notice that the Reeb vector field is not Killing, otherwise the manifold would be coKähler in view of Remark 3.12 ,

A generalization of this example in higher dimensions is considered in [39.

We close the section by illustrating a general method for defining left invariant coKähler structures on Lie groups. First recall the notion of coKähler Lie algebra. Namely, a coKähler Lie algebra is a real Lie algebra $\mathfrak{g}$ of dimension $2 n+1$ endowed with a 1 -form $\eta$, an endomorphism $\phi$ and an inner product $g$ satisfying the conditions

$$
\begin{gathered}
\eta(\xi)=1, \quad \mathrm{~d} \eta=0, \quad \mathrm{~d} \Phi=0 \\
\phi^{2}=-I+\eta \otimes \xi, \quad N_{\phi}^{(1)}=0, \quad g(\phi \cdot \phi \cdot)=g-\eta \otimes \eta,
\end{gathered}
$$

where $\xi$ is the dual vector field of $\eta, \Phi$ is the 2-form defined by $\Phi:=g(\cdot, \phi \cdot)$ and $N_{\phi}^{(1)}$ is the tensor field formally defined as in (3.2).

This notion is closely related to that of a Kähler Lie algebra, a real Lie algebra of dimension $2 n$ endowed with a 2-form $\omega$ and an endomorphism $J$ satisfying

$$
\mathrm{d} \omega=0, \quad J^{2}=-I, \quad[J, J]=0, \quad g(J \cdot, J \cdot)=g .
$$

Concerning coKähler Lie algebras, Fino and Vezzoni proved the following characterization.

Theorem 3.20 (81]). CoKähler Lie algebras of dimension $2 n+1$ are in one-to-one correspondence with $2 n$-dimensional Kähler Lie algebras equipped with a skew-adjoint derivation $D$ commuting with the complex structure.

It follows in particular that any coKähler unimodular Lie group is necessarily flat and solvable.

Actually, the above result was proved at the Lie group level by Dacko in 45 for almost coKähler manifolds. His results can be summarized as follows.

Theorem $3.21(\sqrt[45]{)})$. Let $(\phi, \xi, \eta, g)$ be a left-invariant almost coKähler structure on a simply connected and connected Lie group $G$. Then $G$ is a semi-direct product of the form $\mathbb{R} \times{ }_{f} G_{1}$, where $G_{1}$ is a Lie group and $f$ is a 1-parameter group of automorphisms of $G_{1}$, and $(\phi, \xi, \eta, g)$ is an almost coKähler extension of some almost Kähler structure on $G_{1}$.

3.6. Curvature of coKähler manifolds. Curvature properties of coKähler manifolds are among the most studied topics in coKähler geometry. We start by collecting some basic properties of almost coKähler manifolds. We adopt the following notation. We denote by $R$ the curvature tensor defined by $R(X, Y, Z, W):=-g(R(X, Y) Z, W)$, by Ric the Ricci tensor, given by $\operatorname{Ric}(X, Y):=\sum_{i=0}^{2 n} R\left(E_{i}, X, Y, E_{i}\right)$, where $\left\{E_{0}:=\xi, E_{1}, \ldots, E_{2 n}\right\}$ is a local orthonormal basis. Furthermore we denote by Ric ${ }^{*}$ the Ricci $*-$ tensor, defined by $\operatorname{Ric}^{*}(X, Y):=\sum_{i=0}^{2 n} R\left(E_{i}, X, \phi Y, \phi E_{i}\right)$, and, similarly, $s^{*}$ will be the scalar $*-$ curvature, given by the trace of Ric*. Moreover we shall denote by $K$ the sectional curvature, defined by $K(\pi)=$ $K(X, Y):=R(X, Y, X, Y)$, where $X$ and $Y$ are orthonormal vectors spanning the 2-plane $\pi \subset T_{x} M$. 
The first systematic study of the curvature of an almost coKähler manifold is due to Olszak ([125]). He found useful formulas relating the curvature tensor of an almost coKähler manifold with other tensor fields, such as the covariant derivative of $\phi$, etc.

Theorem 3.22 ([125]). In any almost coKähler manifold the following identities hold

$$
\begin{gathered}
R(\xi, X) \xi-\phi R(\xi, \phi X) \xi+2 h^{2} X=0, \\
s-s^{*}-\operatorname{Ric}(\xi, \xi)+\frac{1}{2}\|\nabla \phi\|^{2}=0, \\
\operatorname{Ric}(\xi, \xi)+\|\nabla \xi\|^{2}=0,
\end{gathered}
$$

where $h=\frac{1}{2} \mathcal{L}_{\xi} \phi$. Furthermore, any compact almost contact metric manifold $(M, \phi, \xi, \eta, g)$ satisfying (3.14) and (3.15) is necessarily almost coKähler.

A variation of the last sufficient condition is the following.

Theorem 3.23 (125). Let $(M, \phi, \xi, \eta, g)$ be an almost contact metric manifold such that the integral curves of the Reeb vector field are geodesics and the forms $\eta$ and $\Phi$ are coclosed. If $M$ fulfills the conditions (3.14) and (3.15), then it is almost coKähler.

Now, one can prove $(125)$ that in any 3 -dimensional almost contact metric manifold one has $\|\nabla \phi\|^{2}=$ $2\|\nabla \xi\|^{2}, 3\|\mathrm{~d} \Phi\|^{2}=2(\delta \eta)^{2}$ and $\|\delta \Phi\|^{2}=2\|\mathrm{~d} \eta\|^{2}$. Thus we can state the following theorems.

Theorem 3.24 ([125]). Any 3-dimensional almost contact metric manifold is coKähler if and only if the Reeb vector field is parallel.

Theorem 3.25 ([125]). Let $(M, \phi, \xi, \eta, g)$ be a 3 -dimensional almost contact metric manifold such that any among the following conditions is satisfied:

(i) At least one among $\eta$ or $\Phi$ is a harmonic form.

(ii) $M$ is compact and (3.15) holds.

Then $M$ is almost coKähler.

Blair proved that a coKähler manifold of constant curvature is locally flat ([12]). Later on, Goldberg and Yano proved in 90, that an almost coKähler manifold of constant curvature is coKähler if and only if it is locally flat. Finally, Olszak ([125, [127]) showed that almost coKähler manifolds of non-zero constant curvature do not exist in any dimension. Summing up, we can state the following important theorem.

Theorem 3.26. If an almost coKähler manifold $M$ is of constant curvature, then the metric is flat and the structure is coKähler.

Turning to coKähler manifolds, we state the following properties.

Theorem 3.27 ([90]). In any coKähler manifold $(M, \phi, \xi, \eta, g)$ the following properties hold

(i) $R(\phi X, \phi Y)=R(X, Y)$,

(ii) $K(\phi X, \phi Y)=K(X, Y)$,

(iii) $g(R(X, \phi X) Y, \phi Y)=-K(X, Y)-K(X, \phi Y)$, where $X$ and $Y$ are orthonormal vector fields.

From Theorem 3.27 it follows that

$$
\operatorname{Ric}(\phi X, \phi Y)=\operatorname{Ric}(X, Y)
$$

for any $X, Y \in \Gamma(T M)$. In particular we have that $\operatorname{Ric}(X, \xi)=\operatorname{Ric}(\phi X, \phi \xi)=0$ and $\operatorname{Ric}(X, \phi Y)=$ $\operatorname{Ric}(\phi X,-Y+\eta(Y) \xi)=-\operatorname{Ric}(\phi X, Y)$. This last property implies that the tensor $\rho(X, Y)=\operatorname{Ric}(X, \phi Y)$ is in fact a 2 -form. 
By putting $Y=\xi$ in (i) and (ii) of Theorem 3.27, we get, respectively,

$$
R(X, \xi)=0, \quad K(X, \xi)=0
$$

for any $X \in \Gamma(T M)$. Moreover, as an immediate consequence of Theorem 3.9, one has that

$$
R(X, Y) \phi Z=\phi R(X, Y) Z
$$

for any vector fields $X, Y, Z$. An important result due to Goldberg and Yano ensures that the converse also holds.

Theorem 3.28 (90]). If the curvature transformation of the metric $g$ of the almost coKähler manifold $(M, \phi, \xi, \eta, g)$ commutes with $\phi$, then $M$ is normal, that is, it is a coKähler manifold.

From (3.17) it easily follows that

$$
R(X, Y) \xi=0 .
$$

Actually (3.18) holds true for any almost coKähler manifold with $\xi$ Killing, since $\nabla \xi=0$. We now prove that this is in fact a characterization of this class of almost coKähler manifolds.

Theorem 3.29. Let $(M, \phi, \xi, \eta, g)$ be an almost coKähler manifold. Then the Reeb vector field $\xi$ is Killing if and only if $R(X, Y) \xi=0$ for any $X, Y \in \Gamma(T M)$.

Proof. Clearly if $\xi$ is Killing then $R(X, Y) \xi=0$, as pointed out before. Conversely, let us assume that $R(X, Y) \xi=0$ for any $X, Y \in \Gamma(T M)$. Then in particular one has $R(\xi, X) \xi=0$, where $X$ in any vector field orthogonal to $\xi$. Then by applying (3.13) we get $h^{2} X=0$. This in turn implies, since $g$ is positive definite, that $h X=0$. As $h \xi=0$, we conclude that the tensor field $h$ vanishes identically, so that, by Theorem 3.11, $\xi$ is a Killing vector field.

Corollary 3.30. In any almost coKähler manifold with $\xi$ Killing one has Ric $^{\sharp} \xi=0$, where Ric ${ }^{\sharp}$ denotes the Ricci operator.

Now, let $X, Y, Z$ any vector fields on a coKähler manifold $(M, \phi, \xi, \eta, g)$ and decompose $Z$ as $Z=Z_{\mathcal{D}}+$ $\eta(Z) \xi$, according to the orthogonal decomposition $T M=\mathcal{D} \oplus \mathbb{R} \xi$. Then, since $\nabla \mathcal{D} \subset \mathcal{D}, \eta(R(X, Y) Z)=$ $\eta\left(R(X, Y) Z_{\mathcal{D}}\right)+\eta(Z) \eta(R(X, Y) \xi)=0$. Using this, (3.17) and (i) of Theorem 3.27, we obtain

$$
R(\phi X, \phi Y, \phi Z, \phi W)=R(X, Y, Z, W)
$$

for all $X, Y, Z, W \in \Gamma(T M)$. Using (3.19) one proves the following theorem (cf. 68]).

Theorem 3.31. On any coKähler manifold the Ricci operator Ric ${ }^{\sharp}$ commutes with $\phi$.

Proof. Let us consider a $\phi$-basis $\left\{E_{1}, \ldots, E_{2 n}, \xi\right\}$, where for each $i \in\{1, \ldots, n\} E_{n+i}=\phi E_{i}$. Then, for any $X, Y \in \Gamma(T M)$,

$$
\begin{aligned}
g\left(\operatorname{Ric}^{\sharp} \phi X, \phi Y\right) & =\sum_{i=1}^{2 n} R\left(E_{i}, \phi X, \phi Y, E_{i}\right)+R(\xi, \phi X, \phi Y, \xi) \\
& =\sum_{i=1}^{2 n} R\left(\phi E_{i}, \phi X, \phi Y, \phi E_{i}\right)-g(R(\xi, \phi X) \phi Y, \xi) \\
& =\sum_{i=1}^{2 n} R\left(E_{i}, X, Y, E_{i}\right)+R(\xi, X, Y, \xi) \\
& =g\left(\operatorname{Ric}^{\sharp} X, Y\right) .
\end{aligned}
$$


By Corollary 3.30, $\operatorname{Ric}^{\sharp} \xi=0$, so that $\eta\left(\operatorname{Ric}^{\sharp} X\right)=g\left(\operatorname{Ric}^{\sharp} X, \xi\right)=g\left(\operatorname{Ric}^{\sharp} \xi, X\right)=0$. Hence we have $-g\left(\phi \operatorname{Ric}^{\sharp} \phi X, Y\right)=g\left(\operatorname{Ric}^{\sharp} X, Y\right)$, from which it follows that

$$
\phi \operatorname{Ric}^{\sharp} \phi X=-\operatorname{Ric}^{\sharp} X .
$$

Applying $\phi$ yields $\phi \operatorname{Ric}^{\sharp} X=-\phi^{2} \operatorname{Ric}^{\sharp} \phi X=\operatorname{Ric}^{\sharp} \phi X-\eta\left(\operatorname{Ric}^{\sharp} \phi X\right) \xi=\operatorname{Ric}^{\sharp} \phi X$.

Theorem 3.26 shows that the requirement of constancy of the sectional curvature is too strong for a coKähler manifold. This leads us to introduce the notion of $\phi$-sectional curvature (also called $\phi$ holomorphic curvature). Given a manifold $M$ with coKähler structure $(\phi, \xi, \eta, g)$, we call a $\phi$-section any plane section of $T_{x} M$ spanned by $\{X, \phi X\}$, where $X$ is a vector orthogonal to $\xi$. The sectional curvature $H(X):=K(X, \phi X)$ corresponding to such a section is then called $\phi$-sectional curvature. Eum ([72], see also [113]) proved the following important theorem

Theorem 3.32. If a coKähler manifold has a constant $\phi$-sectional curvature at every point, then the curvature tensor $R$ of the manifold is given by

$$
\begin{aligned}
R(X, Y) Z= & \frac{c}{4}(g(X, Z) Y-g(Y, Z) X+g(\phi X, Z) \phi Y-g(\phi Y, Z) \phi X \\
& +2 g(\phi X, Y) \phi Z-\eta(X) \eta(Z) Y+\eta(Y) \eta(Z) X+(\eta(X) g(Y, Z)-\eta(Y) g(X, Z)) \xi),
\end{aligned}
$$

where $c$ is constant.

CoKähler manifolds with constant $\phi$-sectional curvature are usually referred as coKähler space forms $M(c)$. There are several papers dealing with coKähler space forms, in many different contexts. We mention the result due to de León and Marrero that given a $(2 n+1)$-dimensional compact coKähler manifold $M$ with positive constant $\phi$-sectional curvature $c$, there exists a diffeomorphism $f: M \longrightarrow P_{n}(\mathbb{C})(c) \times S^{1}$, of $M$ onto the product of $S^{1}$ with the complex projective space of positive constant holomorphic sectional curvature $c$, which is transversally holomorphic isometric. Recall that a diffeomorphism $f: M \longrightarrow M^{\prime}$ between two coKähler manifolds is said to be transversally holomorphic isometric if $f^{*} \eta^{\prime}=\eta$ and for all $x \in M$ the mapping $\left.f\right|_{L_{x}}: L_{x} \longrightarrow L_{f(x)}^{\prime}$ is a Hermitian isometry between the Kähler manifolds $L_{x}$ and $L_{f(x)}^{\prime}$, where $L_{x}$ is the leaf of $\mathcal{D}$ passing through $x$ and where $L_{f(x)}^{\prime}$ is the leaf of $\mathcal{D}^{\prime}$ over $f(x)$.

Remark 3.33. Notice that coKähler space forms sit inside the more general framework of generalized Sasakian-space-forms (see 2 for more details).

By using (3.20), one can prove that the Ricci tensor of a coKähler space form $M(c)$ has the following expression

$$
\operatorname{Ric}(X, Y)=\frac{n+1}{2} c(g(X, Y)-\eta(X) \eta(Y)) .
$$

More in general, if the Ricci tensor of an almost coKähler manifold has the form

$$
\text { Ric }=a g+b \eta \otimes \eta,
$$

where $a$ and $b$ are smooth functions, we say that $M$ is $\eta$-Einstein. In particular if the Reeb vector field is Killing, by Corollary 3.30 we get $a+b=0$. Notice that if $\xi$ is is Killing and the dimension $2 n+1 \geq 5$ then $a$ and $b$ are necessarily constants. Thus we conclude that any almost coKähler manifold such that the Reeb vector field is Killing is Einstein if and only if it is Ricci-flat. Regarding to this, we mention the following result, which can be considered as an odd-dimensional counterpart of the famous Goldberg conjecture.

Theorem 3.34 (34). Every compact Einstein almost coKähler manifold with Killing Reeb vector field is necessarily coKähler.

Corollary 3.35. Any compact Ricci-flat almost coKähler manifold is coKähler. 
It follows that if $(M, \phi, \xi, \eta, g)$ is a compact coKähler-Einstein manifold, then any other almost coKähler structure $\left(\phi^{\prime}, \xi^{\prime}, \eta^{\prime}, g\right)$, compatible with the same metric $g$, is necessarily normal. Indeed since $(M, g)$ is coKähler-Einstein, it is Ricci-flat. Then by (3.15) $\left\|\nabla \xi^{\prime}\right\|=0$ and so $\xi^{\prime}$ is Killing. The assertion then follows from Theorem 3.34 applied to $\left(\phi^{\prime}, \xi^{\prime}, \eta^{\prime}, g\right)$.

Concerning $\eta$-Einstein manifolds, de León and Marrero proved the following result.

Theorem 3.36 (56]). Every compact $\eta$-Einstein almost coKähler manifold $M$ such that the Einstein constant $a>0$ and $\xi$ is a Killing vector field is a coKähler manifold. Furthermore, $M$ is locally a product of a simply connected compact Kähler-Einstein manifold and $S^{1}$, and $\pi_{1}(M)$ is isomorphic to $\mathbb{Z}$.

We close the section by mentioning an important generalization of the condition $R(X, Y) \xi=0$ that characterizes almost coKähler manifolds with Killing Reeb vector field.

Namely, given an almost contact metric manifold $(M, \phi, \xi, \eta, g)$ and two real numbers $\kappa$ and $\mu$ one can define a distribution $N(\kappa, \mu)$ on $M$ by

$$
N_{x}(\kappa, \mu):=\left\{Z \in T_{x} M \mid R(X, Y) Z=\kappa(g(Y, Z) X-g(X, Z) Y)+\mu(g(Y, Z) h X-g(X, Z) h Y)\right\} .
$$

The distribution $N(\kappa, \mu)$ is called the $(\kappa, \mu)$-nullity distribution. In [17, the case when the Reeb vector field of a contact metric manifold belongs to the $(\kappa, \mu)$-nullity distribution was considered. A few years later, the almost coKähler case was also considered ([69]).

Thus an almost coKähler $(\kappa, \mu)$-manifold is an almost coKähler manifold satisfying the curvature condition

$$
R(X, Y) \xi=\kappa(\eta(Y) X-\eta(X) Y)+\mu(\eta(Y) h X-\eta(X) h Y),
$$

for some $\kappa, \mu \in \mathbb{R}$. In particular, any coKähler manifold satisfies such a condition for $\kappa=0$ and any $\mu$. However, if $\kappa \neq 0$ any almost coKähler $(\kappa, \mu)$-manifold cannot be coKähler, because of the relation (see 69])

$$
h^{2}=\kappa \phi^{2} .
$$

Notice also that from (3.22) it follows that $\kappa \leq 0$ and $\kappa=0$ if and only if $M$ is coKähler. In the sequel we shall consider the non-coKähler case, that is $\kappa<0$. In this case one proves that the operator $h$ admits 3 distinct eigenvalues, 0 and $\pm \lambda:= \pm \sqrt{-\kappa}$, and the tangent bundle of the manifold splits up as the orthogonal sum of the corresponding eigendistributions $T M=\mathcal{D}_{h}(\lambda) \oplus \mathcal{D}_{h}(-\lambda) \oplus \mathcal{D}_{h}(0)$, where $\mathcal{D}_{h}(0)=\mathbb{R} \xi$. Moreover, $\mathcal{D}_{h}(\lambda)$ and $\mathcal{D}_{h}(-\lambda)$ are totally geodesic foliations and are related by $\phi \mathcal{D}_{h}( \pm \lambda)=\mathcal{D}_{h}(\mp \lambda)$. This decomposition allows us to prove that the condition (3.21) determines the curvature completely (cf. [69, Theorem 3.1]). In particular it follows that if $X$ is a unit vector field in $\mathcal{D}_{h}(\lambda)$ one has

$$
K(X, \xi)=\kappa+\lambda \mu, \quad K(\phi X, \xi)=\kappa-\lambda \mu, \quad K(X, \phi X)=-\kappa .
$$

Furthermore, an explicit formula for the Ricci operator is provided

$$
\operatorname{Ric}^{\sharp}=\mu h+2 n \kappa \eta \otimes \xi .
$$

Remark 3.37. From (3.23) it follows that $\operatorname{Ric}^{\sharp} \xi=2 n \kappa \xi$. On the other hand, as proved in [129] (see also page 214 of [63]), Theorem 3.7 implies that the Reeb vector field of an almost coKähler manifold is a harmonic vector field if and only if it is an eigenvector of the Ricci operator. In view of this remark and Corollary 3.30, it follows that in any almost coKähler manifold such that $\xi$ is Killing or belongs to the $(\kappa, \mu)$-nullity distribution, the Reeb vector field is harmonic.

Thus, in view of (3.23), almost coKähler $(\kappa, \mu)$-manifolds are $\eta$-Einstein if and only if $\mu=0$, that is

$$
R(X, Y) \xi=\kappa(\eta(Y) X-\eta(X) Y) .
$$

This case was studied, among others, by Dacko (46]), who proved that $M$ is necessarily an almost coKähler manifold with Kählerian leaves and, moreover, gave a full description of the local structure of 
this class. We briefly recall his construction, referring to [46] for more details. Let $\lambda$ be a real positive number and $\mathfrak{g}_{\lambda}$ be the solvable non-nilpotent Lie algebra with basis $\left\{\xi, X_{1}, \ldots, X_{n}, Y_{1}, \ldots, Y_{n}\right\}$ and nonzero Lie brackets

$$
\left[\xi, X_{i}\right]=-\lambda X_{i}, \quad\left[\xi, Y_{i}\right]=\lambda Y_{i}
$$

for each $i \in\{1, \ldots, n\}$. Let $G_{\lambda}$ be a Lie group whose Lie algebra is $\mathfrak{g}_{\lambda}$ and let $(\phi, \xi, \eta, g)$ be the leftinvariant almost coKähler structure defined by putting at the identity

$$
\begin{gathered}
g\left(X_{i}, X_{j}\right)=g\left(Y_{i}, Y_{j}\right)=\delta_{i j}, \quad g\left(X_{i}, Y_{j}\right)=0, \quad g\left(\xi, X_{i}\right)=g\left(\xi, Y_{i}\right)=0 \\
\phi \xi=0, \quad \phi X_{i}=Y_{i}, \quad \phi Y_{i}=-X_{i}, \quad \eta=g(\cdot, \xi) .
\end{gathered}
$$

Then one can prove that the almost coKähler Lie group $\left(G_{\lambda}, \phi, \xi, \eta, g\right)$ satisfies (3.24), with $\kappa=-\lambda^{2}$. Dacko proved that locally all almost coKähler $(\kappa, 0)$-manifolds are of this type:

Theorem $3.38([4])$. Let $(M, \phi, \xi, \eta, g)$ be an almost coKähler manifold whose Reeb vector field $\xi$ satisfies (3.24), for some $\kappa<0$. Then $M$ is locally isomorphic to the above Lie group $G_{\lambda}$ endowed with the almost coKähler structure (3.25), where $\lambda=\sqrt{-\kappa}$.

It remains an open question whether it is possible to provide a full classification as in the contact metric case (cf. [18] and 29]).

Finally, a couple of interesting generalizations of almost coKähler $(\kappa, \mu)$-manifolds are worth mentioning. The first is due to Dacko and Olszak ([52]), who defined an almost coKähler $(\kappa, \mu, \nu)$-manifold as an almost coKähler manifold satisfying the following curvature condition

$$
R(X, Y) \xi=\kappa(\eta(Y) X-\eta(X) Y)+\mu(\eta(Y) h X-\eta(X) h Y)+\nu(\eta(Y) \phi h X-\eta(X) \phi h Y)
$$

where $\kappa, \mu, \nu$ belong to the subring of the ring of smooth functions $f$ on $M$ such that $d f \wedge \eta=0$. Of course, taking $\nu=0$ and $\kappa$ and $\mu$ constant, one reobtains the aforementioned notion of almost coKähler $(\kappa, \mu)$-manifolds. Among other results, the authors proved that the leaves of the canonical foliations $\mathcal{D}$ are locally flat Kählerian manifolds, and then they found local models for the case $\kappa=1$ and $\nu=0$ ([51]).

Very recently, Carriazo and Martín-Molina provided an explicit description of the curvature tensor of an almost coKähler $(\kappa, \mu, \nu)$-space and proposed a further generalization (see [36] for more details).

3.7. Topology of compact coKähler manifolds. An interesting topic is the study of topological properties of coKähler and almost coKähler manifolds. In particular, it is important to investigate the topological obstructions to their existence.

To begin with, recall the result of Goldberg and Yano (Theorem [3.7) that for any almost coKähler manifold the 1-form $\eta$ and the 2-form $\Phi$ are harmonic. In view of Theorem 3.7. it follows that the first and the second Betti number of any compact almost coKähler manifold are different from zero. Actually, as an application of the formula at page 109 of [87, one can prove that in any almost contact metric manifold such that $\xi$ is Killing and $\eta$ is harmonic, the wedge product by $\eta$ preserves harmonicity. It follows that in any compact almost coKähler manifold that satisfies any of the equivalent conditions of Theorem 3.11, the 3-form $\eta \wedge \Phi$ is harmonic and hence also the third Betti number is non-zero.

In fact if the structure is also normal, a stronger result holds.

Theorem 3.39 ([16]). All the Betti numbers of a compact coKähler manifold are non-zero.

In order to prove Theorem 3.39 , one considers the operators $L$ and $\Lambda$, dual to each other, defined by $L \alpha:=\alpha \wedge \Phi$ and $\Lambda \alpha:=\star L \star \alpha$, for any form $\alpha$, where $\star$ denotes the Hodge star isomorphism. In the case when $M$ is coKähler, Blair and Goldberg ([16]) proved that $L$ commutes with the Laplace-Beltrami operator $\Delta:=d \delta+\delta d$. Using this, Theorem 3.39 follows.

Several years later, Chinea, de León and Marrero wrote a fundamental paper on the topology of compact coKähler manifolds $([40])$. An important role in their study is played by the subspaces $\Omega_{H \xi}^{p}(M)$ 
and $\Omega_{H \eta}^{p}(M)$ of the vector space $\Omega_{H}^{p}(M)$ of harmonic $p$-forms, on a compact coKähler manifold $M$ of dimension $2 n+1$, defined by

$$
\begin{gathered}
\Omega_{H \xi}^{p}(M)=\left\{\alpha \in \Omega_{H}^{p}(M) \mid i_{\xi} \alpha=0\right\} \\
\Omega_{H \eta}^{p}(M)=\left\{\alpha \in \Omega_{H}^{p}(M) \mid \eta \wedge \alpha=0\right\} .
\end{gathered}
$$

Then, the authors of [40] prove the splitting

$$
\Omega_{H}^{p}(M)=\Omega_{H \xi}^{p}(M) \oplus \Omega_{H \eta}^{p}(M),
$$

and, moreover, that the dimensions of the above subspaces are topological invariants of $M$.

Using this and the Poincaré duality, one obtains the following monotonicity property.

Theorem 3.40 (40]). On a compact coKähler manifold one has

$$
b_{0} \leq b_{1} \leq \ldots \leq b_{n}=b_{n+1} \geq b_{n+2} \geq \ldots \geq b_{2 n+1} .
$$

Next, by studying complex harmonic forms, Chinea, de León and Marrero prove the coKähler counterpart of the well-known property that the odd-dimensional Betti numbers of a compact Kähler manifold are even.

Theorem 3.41 ([40]). On a compact coKähler manifold one has:

(i) For any odd integer $p$, the dimension of $\Omega_{H \xi}^{p}(M)$ is even.

(ii) For any $0 \leq p \leq n$, the difference $b_{2 p+1}-b_{2 p}$ is even. In particular, the first Betti number of $M$ is odd.

Moreover, in [40] an odd-dimensional version of the Hard Lefschetz Theorem is given. The authors first prove that the linear map $L^{n-p}: \Omega_{H \xi}^{p}(M) \longrightarrow \Omega_{H \xi}^{2 n-p}(M), \alpha \longmapsto \alpha \wedge \Phi^{n-p}$, is an isomorphism. Using this and the decomposition (3.27), they proved the following coKähler version of the Hard Lefschetz Theorem:

Theorem 3.42 (40]). In any compact coKähler manifold, for each $p \leq n$ the linear map of the space $\Omega_{H}^{p}(M)$ into the space $\Omega_{H}^{2 n+1-p}(M)$ given by

$$
\alpha \in \Omega_{H}^{p}(M) \longmapsto i_{\xi}(\alpha \wedge \Phi) \wedge \Phi^{n-p}+\eta \wedge \alpha \wedge \Phi^{n-p} \in \Omega_{H}^{2 n+1-p}(M)
$$

is an isomorphism.

A $p$-form is said to be effective if it belongs to the kernel of $\Lambda=\star L \star$. This definition can be given for any almost contact metric manifold. Next, denoting by $\bar{\Omega}_{H}^{p}(M)$ the space of harmonic effective $p$ forms and putting $\bar{\Omega}_{H \xi}^{p}(M):=\bar{\Omega}_{H}^{p}(M) \cap \Omega_{H \xi}^{p}(M), \bar{\Omega}_{H \eta}^{p}(M):=\bar{\Omega}_{H}^{p}(M) \cap \Omega_{H \eta}^{p}(M)$, one has the following theorem, relating such subspaces with the Betti numbers of $M$.

Theorem 3.43 (40]). On a compact coKähler manifold one has

$$
\operatorname{dim} \bar{\Omega}_{H \xi}^{p}(M)=b_{p}-b_{p-1}, \quad \operatorname{dim} \bar{\Omega}_{H \eta}^{p}(M)=b_{p-1}-b_{p-2},
$$

for any $p \leq n+1$. Thus the dimensions of $\bar{\Omega}_{H \xi}^{p}(M)$ and of $\bar{\Omega}_{H \eta}^{p}(M)$ are topological invariants.

Many of the results in [40] have been revisited in 2008 by Li (107]) using different topological techniques. His work is based on the notion of "symplectic / Kähler mapping torus". We recall the precise definitions. Let $f \in \operatorname{Diff}(S)$ be a diffeomorphism on a closed, connected manifold $S$. The mapping torus $S_{f}$ is obtained from $S \times[0,1]$ by identifying the two ends via $f$, namely

$$
S_{f}=S \times[0,1] /\{(x, 0) \sim(f(x), 1) \mid x \in S\} .
$$

It is known that $S_{f}$ is the total space of a fiber bundle over $S^{1}$ with fiber $S$. Now, if $(S, \Omega)$ is a symplectic manifold and $f$ a symplectomorphism, $S_{f}$ is called symplectic mapping torus. If $(S, J, G)$ is a Kähler manifold and $f$ an Hermitian isometry, we say that $S_{f}$ is a Kähler mapping torus.

The main result of $\mathrm{Li}$ was the following remarkable criteria for the existence of cosymplectic and coKähler structures in terms of mapping tori. 
Theorem 3.44 ([107]). Let $M$ be a closed manifold. Then we have:

(i) $M$ admits a cosymplectic structure if and only there exists a symplectic manifold $(S, \Omega)$ and a symplectomorphism $f$ of $(S, \Omega)$ such that $M$ is diffeomorphic to $S_{f}$.

(ii) $M$ admits a coKähler structure if and only there exists a Kähler manifold $(S, J, G)$ and a Hermitian isometry $f$ of $(S, J, G)$ such that $M$ is diffeomorphic to $S_{f}$.

Theorem 3.44 can be regarded as an extension to the cosymplectic and coKähler settings of the wellknown result due to Tischler that any compact manifold admitting a non-vanishing closed 1-form fibres over a circle (143]). Moreover, by using the second part of Theorem 3.44, Li was able to give a simpler proof of the aforementioned monotonicity of the Betti numbers of a compact coKähler manifold (Theorem 3.40).

A key point in the proof of Theorem 3.44 (as well as in the proof of Tischler's theorem) is that one can approximate the 1-form $\eta$ with a 1-form $\eta_{\theta}$ representing an integral cohomology class. This aspect is used in a very recent paper of Bazzoni and Oprea (9]), where the authors, using Li's characterization, give another type of structure theorem for coKähler manifolds. Namely, they prove the following theorem.

Theorem 3.45 (9]). A compact coKähler manifold $(M, \phi, \xi, \eta, g)$ with integral 1-form $\eta$ and mapping torus bundle $S \hookrightarrow M \longrightarrow S^{1}$ splits as

$$
M \cong S^{1} \times_{\mathbb{Z}_{m}} S,
$$

where $S^{1} \times S \longrightarrow M$ is a finite cover with structure group $\mathbb{Z}_{m}$ acting diagonally and by translation on the first factor. Moreover, $M$ fibres over the circle $S^{1} /\left(\mathbb{Z}_{m}\right)$ with finite structure group.

Using Theorem 3.45 Bazzoni and Oprea are able to recover, in a very simple way, some of the Chinea, de León, Marrero results on the Betti numbers of compact coKähler manifolds. Moreover, they obtain a result regarding the fundamental group of a coKähler manifold. As we remarked, the fundamental group of any coKähler (actually, almost coKähler) manifold is infinite. In fact we can say more:

Theorem 3.46 (9]). If $(M, \phi, \xi, \eta, g)$ is a compact coKähler manifold with integral 1-form $\eta$ and splitting $M \cong S^{1} \times_{\mathbb{Z}_{m}} S$, then $\pi_{1}(M)$ has a subgroup of the form $H \times \mathbb{Z}$, where $H$ is the fundamental group of a compact Kähler manifold, such that the quotient

$$
\frac{\pi_{1}(M)}{H \times \mathbb{Z}}
$$

is a finite cyclic group.

Theorem 3.46] is one of the few results concerning the fundamental group of a coKähler manifold. We mention another one, due to de León and Marrero. They studied in [56] how some curvature conditions can affect the topology of a compact coKähler manifold. In particular they were looking for an odddimensional analogue of the well-known fact that a compact Kähler manifold with positive definite Ricci tensor is simply connected. However, a compact simply connected manifold cannot admit a coKähler structure and, moreover, because of (3.15), the Ricci tensor of a coKähler manifold cannot be positive definite. In particular, the Ricci tensor Ric of a coKähler manifold of positive constant $\phi$-sectional curvature is transversally positive definite, that is for each point $x$ of the manifold $\mathrm{Ric}_{x}$ is positive definite on $\mathcal{D}_{x}([56])$. Now, we have the following theorem.

Theorem 3.47 (56]). Let $M$ be a compact coKähler manifold with transversally positive definite Ricci tensor. Then the space $\Omega_{H \xi}^{1}(M)$ is trivial. Moreover, $b_{1}=1$ and $\pi_{1}(M)$ is isomorphic to $\mathbb{Z}$. In particular, the first integral homology group of $M$ is also isomorphic to $\mathbb{Z}$.

The $\pi_{1}$ part of Theorem 3.47 was recently reproved in [9] using Li's approach. Finally, concerning the almost coKähler case, we mention the following result. 
Theorem 3.48 ([56]). Let $M$ be a compact almost coKähler manifold such that $b_{1}=1$ and $\xi$ is Killing. Then the following statements hold:

(i) If $L$ is a leaf of the foliation $\mathcal{D}$, then the inclusion map induces a monomorphism $i: \pi_{1}(L) \longrightarrow$ $\pi_{1}(M)$ and the quotient group $\frac{\pi_{1}(M)}{\pi_{1}(L)}$ is isomorphic to $\mathbb{Z}$.

(ii) There exists a fibration $\pi: M \longrightarrow S^{1}$ such that the leaves of $\mathcal{D}$ are the fibres of $\pi$.

(iii) The leaves of $\mathcal{D}$ are compact.

3.8. Coeffective cohomology. We say that a form $\alpha \in \Omega^{p}(M)$ in a cosymplectic manifold $(M, \eta, \omega)$ is coeffective if $\alpha \wedge \omega=0$. Denote by $\mathcal{A}^{p}(M)$ the space of coeffective forms of degree $p$. Then since $\omega$ is closed $\left(\mathcal{A}^{p}(M), \mathrm{d}\right)$ is a subcomplex of the de Rham complex. The cohomology $H^{p}(\mathcal{A}(M))$ of the complex $\left(\mathcal{A}^{p}(M), \mathrm{d}\right)$ is called coeffective cohomology of $M$. It was introduced in 41 .

The coeffective cohomology for cosymplectic manifolds was studied in 41, 76, 78. We write $\widetilde{H}^{p}(M)$ for the subspace of $H^{p}(M)$ of cohomology classes $\beta$ such that $\beta \wedge[\omega]=0$.

Chinea, de León and Marrero proved in [40, 41, that for a compact coKähler manifold $M^{2 n+1}$

$$
\begin{array}{lll}
H^{p}(\mathcal{A}(M)) \cong 0, & & \text { for } p \leq n-1 \\
H^{p}(\mathcal{A}(M)) \cong \widetilde{H}^{p}(M), & & \text { for } p \geq n+2 .
\end{array}
$$

In 78 , the authors construct an example of a compact cosymplectic manifold for which the above result fails, thereby providing an example of a cosymplectic manifold $(M, \eta, \omega)$ that does not admit any coKähler metric.

Let $M$ be a (possibly non-compact) cosymplectic manifold. We can consider an almost coKähler structure on $M$. Denote by $c_{p}$ the dimension of $H^{p}(\mathcal{A}(M))$. It was shown in [78] that for any $p \geq n+2$ one has

$$
b_{p}-b_{p+2} \leq c_{p} \leq b_{p}+b_{p+2} .
$$

In particular, every $c_{p}$ is finite. In the case $M$ is a compact coKähler manifold, $c_{p}$ always attains the lower bound $b_{p}-b_{p+2}, p \geq n+2$. An example of a non-compact almost coKähler manifold $M$ such that $c_{p}=b_{p}+b_{p+2}, p \geq n+2$ was also presented in [78].

It is difficult to compute the coeffective cohomology for a general cosymplectic manifold. In 76 Fernández et al. provided a way to reduce this problem to a purely algebraic one in the following special case. Let $G$ be a connected nilpotent Lie group, $\mathfrak{g}$ its Lie algebra. Suppose that $G$ is equipped with an invariant cosymplectic structure $(\eta, \omega)$ and $\Gamma$ is a discrete subgroup of $G$ such that the space of right cosets $\Gamma \backslash G$ is compact. We can consider the Chevalley-Eilenberg complex $\left(\Omega^{p}\left(\mathfrak{g}^{*}\right)\right.$,d) for $\mathfrak{g}^{*}$ as a subcomplex of left invariant forms in $\left(\Omega^{p}(G), \mathrm{d}\right)$. Denote by $\mathcal{A}^{p}\left(\mathfrak{g}^{*}\right)$ the intersection $\Omega^{p}\left(\mathfrak{g}^{*}\right) \cap \mathcal{A}^{p}(G)$. Then $\left(\mathcal{A}^{p}\left(\mathfrak{g}^{*}\right), \mathrm{d}\right)$ is a subcomplex of $\left(\Omega^{p}\left(\mathfrak{g}^{*}\right), d\right)$. Then

$$
H^{p}\left(\mathcal{A}\left(\mathfrak{g}^{*}\right)\right) \cong H^{p}(\mathcal{A}(\Gamma \backslash G)) .
$$

3.9. Rational homotopy type. For a general and detailed treatment of rational homotopy theory the reader is referred to 74 .

Let $\mathbb{K}$ be a field. A commutative differential graded algebra $(A, \mathrm{~d})$ (CDGA short) over $\mathbb{K}$ is a graded algebra $A=\bigoplus_{k \geq 0} A_{k}$ over $\mathbb{K}$ such that for all $x \in A_{k}$ and $y \in A_{l}$ we have

$$
x y=(-1)^{k l} y x
$$

and d: $A_{k} \rightarrow A_{k+1}$ is a differential, i.e. $d^{2}=0$. An example of real commutative differential graded algebra is given by the de Rham complex $\left(\Omega^{*}(M), \mathrm{d}\right)$ of differential forms on a smooth manifold $M$, with the multiplication given by the wedge product.

A CDGA $(A, \mathrm{~d})$ is directly quasi-isomorphic to a CDGA $(B, \mathrm{~d})$ if there is a homomorphism of graded algebras $f: A \rightarrow B$ such that

$$
H^{k}(f): H^{k}(A) \rightarrow H^{k}(B)
$$


are isomorphisms for all $k \geq 0$. Two CDGAs $(A, \mathrm{~d})$ and $(B, \mathrm{~d})$ are quasi-isomorphic if there is a chain of CDGAs $A=A_{0}, A_{1}, \ldots, A_{r}=B$, such that either $A_{j}$ is directly quasi-isomorphic to $A_{j+1}$ or $A_{j+1}$ is directly quasi-isomorphic to $A_{j}$.

Note that from the theory of Sullivan of minimal models it follows that the chain in the above definition can be always chosen to have $r \leq 2$.

For every CDGA $(A, \mathrm{~d})$ the cohomology algebra $H(A)$ can be considered as a CDGA with the zero differential. A CDGA $(A, \mathrm{~d})$ is called formal if $(A, \mathrm{~d})$ and $(H(A), 0)$ are quasi-isomorphic. A manifold $M$ is called formal if $\left(\Omega^{*}(M), \mathrm{d}\right)$ is a formal CDGA, that is if the cohomology ring of $M$ is quasi-isomorphic to the de Rham algebra of $M$.

In [139] Sullivan constructed for every connected topological space $X$ a CDGA $(A(X), \mathrm{d})$ over $\mathbb{Q}$ such that if $X$ is a manifold then $\left(A(X) \otimes_{\mathbb{Q}} \mathbb{R}, \mathrm{d}\right)$ is quasi-isomorphic to $\left(\Omega^{*}(X), \mathrm{d}\right)$. He also proved that $(A(X), \mathrm{d})$ is formal if and only if $\left(\Omega^{*}(X), \mathrm{d}\right)$ is formal.

We say that two topological spaces $X$ and $Y$ have the same rational homotopy type if there is a finite chain of maps

$$
X \rightarrow Y_{1} \leftarrow Y_{2} \rightarrow Y_{3} \leftarrow \cdots \rightarrow Y
$$

such that the induced maps in rational cohomology are isomorphisms.

Let $G$ be a group and $H_{1}, H_{2}$ two subgroups of $G$. Then $\left[H_{1}, H_{2}\right]$ is the set of elements $h_{1} h_{2} h_{1}^{-1} h_{2}^{-1}$ with $h_{1} \in H_{1}, h_{2} \in H_{2}$. The lower central series of $G$ is defined to be the chain of subgroups in $G$

$$
G \supset G_{1} \supset G_{2} \supset G_{3} \supset \ldots
$$

where $G_{1}:=[G, G]$ and $G_{k}:=\left[G_{k}, G\right]$. The group $G$ is called nilpotent if there is an integer $n$ such that $G_{n}$ is trivial. For example, abelian groups are nilpotent since $[G, G]$ is trivial in this case. On the other hand, non-commutative simple groups are never nilpotent.

Denote by $\varepsilon: \mathbb{Z} G \rightarrow \mathbb{Z}$ the augmentation homomorphism, that is $\varepsilon\left(\sum_{g \in G} c_{g} g\right)=\sum_{g \in G} c_{g}$. Define the augmentation ideal $I(G)$ of $\mathbb{Z} G$ to be the kernel of $\varepsilon$. A left $G$-module $N$ is called nilpotent if there is $n \in \mathbb{N}$ such that $I(G)^{n} N=0$. This notion can also be explained differently. Define the submodules $N_{k} \subset N$ by

$$
N_{0}:=N, \quad N_{k}:=\left\langle x-g x \mid x \in N_{k-1}, g \in G\right\rangle .
$$

Then $N$ is nilpotent if and only if there is $n \in \mathbb{N}$ such that $N_{n}=0$.

Recall, that for every topological space $X$ the fundamental group $\pi_{1}(X)$ acts on the higher homotopy groups $\pi_{k}(X), k \geq 2$. Thus we can consider $\pi_{k}(X)$ as $\pi_{1}(X)$-module for every $k \geq 2$. The topological space $X$ is called a nilpotent topological space if $\pi_{1}(X)$ is a nilpotent group and the modules $\pi_{k}(X)$ are nilpotent $\pi_{1}(X)$-modules.

The following theorem is due to Sullivan.

Theorem 3.49. Two nilpotent topological spaces $X$ and $Y$ with finite Betti numbers have the same rational homotopy type if and only if $(A(X), \mathrm{d})$ and $(A(Y), \mathrm{d})$ are quasi-isomorphic CDGAs over $\mathbb{Q}$.

Thus in the case of a nilpotent topological space $X$ the algebra $(A(X), \mathrm{d})$ contains full information about the rational homotopy type of $X$. In particular, the groups $\pi_{k}(X) \otimes \mathbb{Q}$ can be computed from any CDGA quasi-isomorphic $\left(A(X)\right.$, d). If $M$ is a nilpotent manifold then the groups $\pi_{k}(M) \otimes \mathbb{R}$ can be computed from any CDGA quasi-isomorphic to $(A(M) \otimes \mathbb{R}, d)$ or to $\left(\Omega^{*}(M), d\right)$. In particular, if $M$ is a formal manifold, then the groups $\pi_{k}(M) \otimes \mathbb{R}$ can be computed in purely formal way from $H^{*}(M)$.

In 61, Deligne et al. proved that every compact Kähler manifold is formal. Using this, Chinea, de León and Marrero showed in [40] that every compact coKähler manifold is formal as well. This result is most useful if the fundamental group of the manifold in question is nilpotent. Note that in contrast to the case of Kähler manifolds the fundamental group of a coKähler manifold cannot be trivial. 
In 8. Bazzoni, Fernández and Muñoz presented a way to construct non-formal compact cosymplectic manifolds. The idea is as follows. Let $S$ be a symplectic manifold and $f: S \rightarrow S$ a symplectomorphism such that for some $p>0$ the eigenvalue $\lambda=1$ of

$$
H^{p}(f): H^{p}(S) \rightarrow H^{p}(S)
$$

has multiplicity two. Then by [8, Theorem 13] and by Theorem 3.44 the mapping torus $S_{f}$ is a nonformal cosymplectic manifold. Using this result Bazzoni, Fernández and Muñoz show that for every pair $(m=2 n+1, b)$ such that either $m=3$ and $b \geq 2$ or $m \geq 5$ and $b \geq 1$, there is a non-formal compact cosymplectic manifold $M$ of dimension $m$ such that the first Betti number of $M$ is $b$.

3.10. Cosymplectic 3-structures. When a smooth manifold $M$ is endowed with three distinct almost contact structures $\left(\phi_{1}, \xi_{1}, \eta_{1}\right),\left(\phi_{2}, \xi_{2}, \eta_{2}\right),\left(\phi_{3}, \xi_{3}, \eta_{3}\right)$ related by the following identities

$$
\begin{gathered}
\phi_{\gamma}=\phi_{\alpha} \phi_{\beta}-\eta_{\beta} \otimes \xi_{\alpha}=-\phi_{\beta} \phi_{\alpha}+\eta_{\alpha} \otimes \xi_{\beta}, \\
\xi_{\gamma}=\phi_{\alpha} \xi_{\beta}=-\phi_{\beta} \xi_{\alpha}, \quad \eta_{\gamma}=\eta_{\alpha} \circ \phi_{\beta}=-\eta_{\beta} \circ \phi_{\alpha},
\end{gathered}
$$

for any even permutation $(\alpha, \beta, \gamma)$ of the set $\{1,2,3\}$, we say that $M$ is endowed with an almost contact 3-structure. In this case $M$ has dimension of the form $4 n+3$. This notion was introduced independently by Kuo ([105]) and Udriste (147]) at the end of the 60 s and then it was studied by several authors, especially from the Japanese school of Riemannian geometry. In particular, Kuo proved that one can always find a Riemannian metric $g$ which is compatible with each almost contact structure. If we fix one, we speak of an almost contact metric 3-structure.

Any smooth manifold endowed with an almost contact metric 3-structure carries two orthogonal distributions, not necessarily integrable: the Reeb distribution $\mathcal{V}:=\operatorname{span}\left\{\xi_{1}, \xi_{2}, \xi_{3}\right\}$ and the horizontal distribution $\mathcal{H}:=\operatorname{ker}\left(\eta_{1}\right) \cap \operatorname{ker}\left(\eta_{2}\right) \cap \operatorname{ker}\left(\eta_{3}\right)=\mathcal{V}^{\perp}$.

The most famous class of almost contact metric 3-structures is given by those for which each structure is Sasakian. They are called 3-Sasakian structures (or Sasakian 3-structures). For more details on 3 -Sasakian manifolds see 23 .

When each structure $\left(\phi_{\alpha}, \xi_{\alpha}, \eta_{\alpha}, g\right)$ is (almost) coKähler, $M$ is called an (almost) 3-cosymplectic manifold. However, it has been proved recently (see [73. Theorem 4.13]) that these two notions are the same, i.e. every almost 3-cosymplectic manifold is normal and thus 3-cosymplectic.

Remark 3.50. Actually, the name 3-cosymplectic is not coherent with the terminology that we used in the rest of this survey. A more appropriate name should have been "3-coKähler". However in literature the term 3-cosymplectic is well-established and, so far, we did not find any other name for these structures (except "hypercosymplectic 3-structure" in [138] and [101]). Thus in this section we shall conform to the terminology currently used in literature.

Remark 3.51. We notice that just as in the case of a single structure, the 3-Sasakian and the 3cosymplectic manifolds represent the two extremal cases of the larger class of 3-quasi-Sasakian manifolds (cf. [31, [32]).

In any 3-cosymplectic manifold the Reeb vector fields $\xi_{1}, \xi_{2}, \xi_{3}$ are all $\nabla$-parallel. In particular, for any $\alpha, \beta \in\{1,2,3\}$, we have $\left[\xi_{\alpha}, \xi_{\beta}\right]=\nabla_{\xi_{\alpha}} \xi_{\beta}-\nabla_{\xi_{\alpha}} \xi_{\beta}=0$. Thus the Reeb distribution is integrable and defines a 3-dimensional foliation $\mathcal{F}_{3}$ of $M$. As it was proved in $\underline{30}, \mathcal{F}_{3}$ is a Riemannian and transversely hyper-Kähler foliation with totally geodesic leaves. Using this, one proves that every 3-cosymplectic manifold is Ricci-flat ([30, Corollary 3.10]). Moreover, since $\mathrm{d} \eta_{\alpha}=0$, also the horizontal distribution $\mathcal{H}$ is integrable and hence defines a Riemannian, totally geodesic foliation complementary to $\mathcal{F}_{3}$.

On the other hand, Ishihara proved that the Reeb distribution of a 3-Sasakian manifold is a transversely quaternionic-Kähler foliation (97]). The study of transverse geometry allows to understand the difference

between Sasakian and coKähler structures, as illustrated in Table 1. In fact, while both Sasakian and 
TABLE 1. Transverse geometry

\begin{tabular}{cc|cc}
\multicolumn{2}{c}{$\operatorname{dim}(M)=2 n+1$} & \multicolumn{2}{c}{$\operatorname{dim}(M)=4 n+3$} \\
\hline \hline Sasakian & coKähler & 3 -Sasakian & 3 -cosymplectic \\
$\downarrow$ & $\downarrow$ & $\downarrow$ & $\downarrow$ \\
Kähler & Kähler & quaternionic-Kähler & hyper-Kähler
\end{tabular}

coKähler manifolds are considered as a natural odd-dimensional counterpart of Kähler manifolds, at the level of 3-structures their role differs: 3-cosymplectic manifolds should be considered as the most natural odd-dimensional analogue of hyper-Kähler manifolds, whereas 3-Sasakian structures corresponds to quaternionic-Kähler structures.

The standard example of a compact 3-cosymplectic manifold is given by the torus $\mathbb{T}^{4 n+3}$ with the following structure (cf. [118, p.561]). Let $\left\{\theta_{1}, \ldots, \theta_{4 n+3}\right\}$ be a basis of 1 -forms such that each $\theta_{i}$ is integral and closed. Let us define a Riemannian metric $g$ on $\mathbb{T}^{4 n+3}$ by

$$
g:=\sum_{i=1}^{4 n+3} \theta_{i} \otimes \theta_{i}
$$

For each $\alpha \in\{1,2,3\}$ we define a tensor field $\phi_{\alpha}$ of type $(1,1)$ by

$\phi_{\alpha}=\sum_{i=1}^{n}\left(E_{\alpha n+i} \otimes \theta_{i}-E_{i} \otimes \theta_{\alpha+i}+E_{\gamma n+i} \otimes \theta_{\beta n+i}-E_{\beta n+i} \otimes \theta_{\gamma n+i}\right)+E_{4 n+\gamma} \otimes \theta_{4 n+\beta}-E_{4 n+\beta} \otimes \theta_{4 n+\gamma}$ where $\left\{E_{1}, \ldots, E_{4 n+3}\right\}$ is the dual (orthonormal) basis of $\left\{\theta_{1}, \ldots, \theta_{4 n+3}\right\}$ and $(\alpha, \beta, \gamma)$ is a cyclic permutation of $\{1,2,3\}$. Setting, for each $\alpha \in\{1,2,3\}, \xi_{\alpha}:=E_{4 n+\alpha}$ and $\eta_{\alpha}:=\theta_{4 n+\alpha}$, one can easily check that the torus $\mathbb{T}^{4 n+3}$ endowed with the structure $\left(\phi_{\alpha}, \xi_{\alpha}, \eta_{\alpha}, g\right)$ is 3-cosymplectic.

For the non-compact case, the standard example of 3-cosymplectic manifold is given by $\mathbb{R}^{4 n+3}$ with the structure described in [30, Theorem 4.4].

Both the examples above are the global product of a hyper-Kähler manifold with a 3-dimensional abelian Lie group. Such a property always holds locally (see [33, Proposition 7.1]). Thus it makes sense to ask whether there are examples of 3-cosymplectic manifolds which are not the global product of a hyper-Kähler manifold with a 3-dimensional abelian Lie group. As shown in [33], the answer to this question is affirmative and now we illustrate a procedure for constructing such examples. Let $\left(N, J_{\alpha}, G\right)$ be a compact hyper-Kähler manifold of dimension $4 n$ and $f$ a hyper-Kähler isometry on it. We define an action $\varphi$ of $\mathbb{Z}^{3}$ on $N \times \mathbb{R}^{3}$ by

$$
\varphi\left(\left(k_{1}, k_{2}, k_{3}\right),\left(x, t_{1}, t_{2}, t_{3}\right)\right)=\left(f^{k_{1}+k_{2}+k_{3}}(x), t_{1}+k_{1}, t_{2}+k_{2}, t_{3}+k_{3}\right) .
$$

We then define on the orbit space $M_{f}:=\left(N \times \mathbb{R}^{3}\right) / \mathbb{Z}^{3}$ a 3 -cosymplectic structure in the following way. Let us consider the vector fields $\xi_{\alpha}:=\frac{\partial}{\partial t_{\alpha}}$ and the 1 -forms $\eta_{\alpha}:=d t_{\alpha}$ on $N \times \mathbb{R}^{3}$. Next we define, for each $\alpha \in\{1,2,3\}$, a tensor field $\phi_{\alpha}$ on $N \times \mathbb{R}^{3}$ by putting $\phi_{\alpha} X:=J_{\alpha} X$ for any $X \in \Gamma(T N)$ and $\phi_{\alpha} \xi_{\alpha}:=0$, $\phi_{\alpha} \xi_{\beta}:=\epsilon_{\alpha \beta \gamma} \xi_{\gamma}$, where $\epsilon_{\alpha \beta \gamma}$ denotes the sign of the permutation $(\alpha, \beta, \gamma)$ of $\{1,2,3\}$. Then $\left(\phi_{\alpha}, \xi_{\alpha}, \eta_{\alpha}, g\right)$, $g$ denoting the product metric, defines a 3 -cosymplectic structure on $N \times \mathbb{R}^{3}$. Being invariant under the action $\varphi,\left(\phi_{\alpha}, \xi_{\alpha}, \eta_{\alpha}, g\right)$ descends to a 3-cosymplectic structure on $M_{f}$, which we will denote by the same symbol. By using this general procedure in [33] a non-trivial example of compact 3-cosymplectic manifold is constructed. In fact we consider the hyper-Kähler manifold $\mathbb{T}^{4}=\mathbb{H} / \mathbb{Z}^{4}$ and the hyper-Kähler isometry $f$ induced by the multiplication by $\mathbf{i}$ (the same can be made by using the multiplication by $\mathbf{j}$ and $\mathbf{k}$ ). Then the 7-dimensional manifold $M_{f}:=\left(\mathbb{T}^{4} \times \mathbb{R}^{3}\right) / \mathbb{Z}^{3}$, endowed with the geometric structure described above, is a compact 3-cosymplectic manifold which is not the global product of a compact 4-dimensional hyperKähler manifold $K$ with the flat torus. Indeed one has only two possibilities for a compact 4 -dimensional 
hyper-Kähler manifold: either $K \cong \mathbb{T}^{4}$ or it is a complex K3-surface. In the first case $b_{2}\left(K \times \mathbb{T}^{3}\right)=21$, in the second $b_{2}\left(K \times \mathbb{T}^{3}\right)=25$. However, in [33] it was proved that $b_{2}\left(M_{f}^{7}\right)<21$.

We close the section by collecting some results on the topology of compact 3-cosymplectic manifolds. Every 3-cosymplectic manifold is, in particular, coKähler, so that all the results in $\S 3.7$ hold. However the rigidity given by the relations (3.28) connecting the almost contact structures forces the topology of a 3-cosymplectic manifold to satisfy the following more restrictive properties.

Let $b_{k}^{h}$ denote the $k$-th basic Betti number with respect to the foliation $\mathcal{F}_{3}$, that is the dimension of the vector space

$$
\Omega_{H B}^{k}(M)=\left\{\omega \in \Omega_{H}^{k}(M) \mid i_{\xi_{\alpha}} \omega=0 \text { for any } \alpha=1,2,3\right\} .
$$

Theorem 3.52 (33). Let $M$ be a compact 3-cosymplectic manifold of dimension $4 n+3$. Then the $k$-th Betti numbers of $M$ are given by

$$
b_{k}=b_{k}^{h}+3 b_{k-1}^{h}+3 b_{k-2}^{h}+b_{k-3}^{h},
$$

where we assume that $b_{p}^{h}=0$ for $p<0$.

By using (3.29) one proves the following theorem.

Theorem 3.53 (33]). Let $M$ be a compact 3-cosymplectic manifold of dimension $4 n+3$. Then the following statements hold.

(i) Any odd-dimensional basic Betti number $b_{2 k+1}^{h}$ is divisible by 4 .

(ii) For any integer $k, b_{k-1}+b_{k}$ is divisible by 4 .

In 33, we also proved certain inequalities that the Betti numbers have to satisfy. First notice that, as the 1 -forms $\eta_{1}, \eta_{2}, \eta_{3}$, the 2 -forms $\Phi_{1}, \Phi_{2}, \Phi_{3}, \eta_{i} \wedge \eta_{j}$, and the 3 -forms $\eta_{1} \wedge \eta_{2} \wedge \eta_{3}, \eta_{i} \wedge \Phi_{j}$, are harmonic and linearly independent, we have $b_{1} \geq 3, b_{2} \geq 6, b_{3} \geq 10$. More generally, we have the following formula.

Theorem $3.54([33])$. Let $M$ be a compact 3 -cosymplectic manifold of dimension $4 n+3$. Then, for any integer $k \in\{0, \ldots, 2 n+1\}$,

$$
b_{k} \geq\left(\begin{array}{c}
k+2 \\
2
\end{array}\right)
$$

Theorem 3.54 should be compared with the corresponding even-dimensional result. Indeed, due to Wakakuwa ([149]), in any compact hyper-Kähler manifold the even Betti number $b_{2 k}(0 \leq k \leq 2 n)$ satisfies

$$
b_{2 k} \geq\left(\begin{array}{c}
k+2 \\
2
\end{array}\right) .
$$

Comparing (3.30) with (3.31), we clearly note that in the 3-cosymplectic case we have a much stronger condition.

We conclude the section by mentioning that the space $\Omega_{H B}^{k}(M)$ of harmonic basic $k$-forms admits an action of the Lie algebra $\mathfrak{s o}(4,1)$. For each $\alpha \in\{1,2,3\}$, let us define the 2 -form

$$
\Xi_{\alpha}:=\frac{1}{2}\left(\Phi_{\alpha}+2 \eta_{\beta} \wedge \eta_{\gamma}\right)
$$

where $(\alpha, \beta, \gamma)$ is an even permutation of $\{1,2,3\}$. Next we define the operators

$$
\begin{gathered}
L_{\alpha}: \Omega^{k}(M) \longrightarrow \Omega^{k+2}(M), \quad L_{\alpha} \omega:=\Xi_{\alpha} \wedge \omega \\
\Lambda_{\alpha}: \Omega^{k+2}(M) \longrightarrow \Omega^{k}(M), \quad \Lambda_{\alpha}:=\star L_{\alpha} \star
\end{gathered}
$$

One can prove that $L_{\alpha}$ and $\Lambda_{\alpha}$ induce endomorphisms of $\Omega_{H B}^{*}(M)$ (which we shall denote by the same symbol). Then, by [33, Proposition 4.3] one has that, on $\Omega_{H B}^{*},\left[L_{\alpha}, \Lambda_{\alpha}\right]=-H$, where $H: \Omega_{H B}^{k}(M) \longrightarrow$ $\Omega_{H B}^{k}(M)$ is the operator defined by $H \omega=(2 n-k) \omega$. Moreover for each $\alpha \in\{1,2,3\}$ we define another 
operator $K_{\alpha}$ on $\Omega_{H B}^{k}(M)$ by $K_{\alpha}:=\left[L_{\beta}, \Lambda_{\gamma}\right]$, where $(\alpha, \beta, \gamma)$ is an even permutation of $\{1,2,3\}$. Then we have the following result.

Theorem 3.55 (33]). The linear span $\mathfrak{g}$ of the operators $H, L_{\alpha}, \Lambda_{\alpha}, K_{\alpha}, \alpha \in\{1,2,3\}$, is a Lie algebra isomorphic to $\mathfrak{s o}(4,1)$. Consequently $\Omega_{H B}^{*}(M)$ is an $\mathfrak{s o}(4,1)$-module.

\section{Further topics}

There are many other topics related to cosymplectic / coKähler geometry. Unfortunately, due to the lack of time and space, we can only give a very brief outline.

4.1. Submanifolds of coKähler manifolds. In almost contact Riemannian geometry there have always been a great interest toward the theory of submanifolds. The most important class of submanifolds of an almost contact metric manifold $(M, \phi, \xi, \eta, g)$ is given by invariant submanifolds.

A submanifold $M^{\prime}$ of $M$ is said to be invariant if $\xi$ is tangent to the submanifold and, for each $x \in M^{\prime}$, $\phi\left(T_{x} M^{\prime}\right) \subset T_{x} M^{\prime}$. Then $M^{\prime}$ inherits an almost contact metric structure $\left(\phi^{\prime}, \xi^{\prime}, \eta^{\prime}, g^{\prime}\right)$ from the ambient space by restriction and $\left(\phi^{\prime}, \xi^{\prime}, \eta^{\prime}, g^{\prime}\right)$ is (almost) coKähler provided that $(\phi, \xi, \eta, g)$ is (almost) coKähler ([113). An important result that invariant submanifolds of almost coKähler manifolds share with contact Riemannian geometry ([15], page 152) is the following, due to Endo.

Theorem 4.1 ([65]). Any invariant submanifold of an almost coKähler manifold is minimal.

Theorem 4.1 suggests to investigate for conditions ensuring that an invariant submanifold of an (almost) coKähler manifold is totally geodesic. In [113] and 89. Ludden and Goldberg, respectively, studied this problem for codimension 2 submanifolds.

In [65, 66], Endo obtained some partial results on invariant submanifolds that we collect in the following theorem.

Theorem 4.2. Let $M^{\prime}$ be an invariant submanifold of an almost coKähler manifold $M$ with pointwise constant $\phi$-sectional curvature $c$. Let $2 m+1$ be the dimension of $M^{\prime}$.

(i) If $M^{\prime}$ has constant $\phi^{\prime}$-sectional curvature $c^{\prime}$ then $c^{\prime} \leq c$, with equality holding if and only if $M^{\prime}$ is totally geodesic.

(ii) The scalar curvature $s^{\prime}$ of $M^{\prime}$ satisfies the inequality $s^{\prime} \leq m(m+1) c$. Moreover, the equality holds if and only if $M^{\prime}$ is totally geodesic and coKähler.

Another remarkable class of submanifolds of an almost contact metric manifold is given anti-invariant submanifolds. A submanifold of an almost contact metric manifold is said to be anti-invariant if $\phi\left(T_{x} M^{\prime}\right) \subset$ $\left(T_{x} M^{\prime}\right)^{\perp}$. A systematic study of anti-invariant submanifolds of coKähler manifolds was developed by Kim 102. Among other result, the author found conditions ensuring that an anti-invariant submanifold of a coKähler manifold is conformally flat, or locally symmetric, or a totally geodesic submanifold.

We just mention also that the notion of invariant and anti-invariant submanifold were generalized in the class of $C R$-submanifold, which was proposed by Bejancu in the context of almost Hermitian geometry and then extended to the almost contact setting. Namely, a submanifold $M^{\prime}$ of an almost coKähler manifold $M$ is called CR-submanifold if $\xi$ is tangent to $M^{\prime}$ and there exists an invariant distribution $D$ whose orthogonal complement $D^{\perp}$ is anti-invariant, i.e. $T M^{\prime}=D \oplus D^{\perp} \oplus \mathbb{R} \xi$ with $\phi D_{x} \subset D_{x}$ and $\phi D_{x}^{\perp} \subset\left(T_{x} M^{\prime}\right)^{\perp}$. Further details can be found in [10] and [151.

The study of the geometry of submanifolds of coKähler manifolds is a current topic of research. There are several papers dealing with the above and other classes of submanifolds of a coKähler manifold (especially coKähler space forms). Here we mention the very recent paper [80] of Fectu and Rosenberg. In that paper, they study the two-dimensional submanifolds with parallel mean curvature in a coKähler space form. 
4.2. Harmonic maps and coKähler geometry. Another interesting topic is the study of the interplay between coKähler geometry with the theory of harmonic maps. Recall that given a smooth map $f$ : $\left(M^{m}, g\right) \longrightarrow\left(M^{\prime}, g^{\prime}\right)$ between Riemannian manifolds, one defines the energy density of $f$ as the smooth function $e(f): M \longrightarrow[0,+\infty)$ given by

$$
e(f)(x)=\frac{1}{2}\left\|f_{* x}\right\|^{2}=\frac{1}{2} \sum_{i=1}^{m} g^{\prime}\left(f_{* x}\left(e_{i}\right), f_{* x}\left(e_{i}\right)\right),
$$

for any $x \in M$, where $\left\{e_{1}, \ldots, e_{m}\right\}$ is any local orthonormal basis of $T_{x} M$. If $M$ is compact, the energy of $f$ is defined by

$$
E(f)=\int_{M} e(f) \nu_{g}
$$

where $\nu_{g}$ is the volume measure associated with the metric $g$ on $M$. Then $f$ will be said to be a harmonic map if it is a critical point of the energy functional $E$ on the set of all maps between $(M, g)$ and $\left(M^{\prime}, g^{\prime}\right)$.

In particular it is of interest to study the harmonicity of the maps to, from and between (almost) coKähler manifolds, especially in the case when one of the two spaces is Kähler. We refer the reader to the papers of Boeckx and Gherghe $([19])$, Chinea ([37]), Gherghe ([84, [85]) and Fectu ([79]).

4.3. Generalizations of cosymplectic and coKähler manifolds. Several generalizations of cosymplectic and coKähler manifolds have been considered. We mention just a few of them, recalling the definitions and referring the interested reader to the references below.

The first generalization that we recall is given by locally conformal cosymplectic manifolds, introduced by Chinea, de León, Marrero in [38] and locally conformal coKähler manifolds, defined by Olszak in [128]. An almost cosymplectic manifold $(M, \eta, \omega)$ is said to be locally conformal cosymplectic (l.c.c.), if there exist an open covering $\left\{U_{i}\right\}_{i \in I}$, and a family of functions $\sigma: U_{i} \longrightarrow \mathbb{R}$ such that $\eta_{i}=\left.e^{\sigma_{i}} \eta\right|_{U_{i}}$ and $\omega_{i}=\left.e^{2 \sigma_{i}} \omega\right|_{U_{i}}$ are closed, i.e. $\left(\eta_{i}, \omega_{i}\right)$ is a cosymplectic structure on $U_{i}$. Then one proves that the local 1-forms $d \sigma_{i}$ glue up to a closed 1-form $\theta$, called the Lee form, which satisfies

$$
\mathrm{d} \eta=\eta \wedge \theta, \quad \mathrm{d} \omega=-2 \omega \wedge \theta .
$$

Clearly, if $(M, \omega, \eta)$ is cosymplectic, then it is l.c.c. with zero Lee form. Analogously one gives the definition of locally conformal coKähler manifolds. Let $(M, \phi, \xi, \eta, g)$ be an almost contact metric manifold. Then $M$ is said to be locally conformal coKähler (l.c.cK.) if there exists an open covering $\left\{U_{i}\right\}_{i \in I}$ of $M$ and a family $\left\{\sigma_{i}\right\}_{i \in I}$ of real-valued smooth functions on each $U_{i}$ such that $\left(U_{i}, \phi_{i}, \xi_{i}, \eta_{i}, g_{i}\right)$ is a coKähler manifold, where

$$
\phi_{i}=\left.\phi\right|_{U_{i}}, \quad \xi_{i}=\left.e^{-\sigma_{i}} \xi\right|_{U_{i}}, \quad \eta_{i}=\left.e^{\sigma_{i}} \eta\right|_{U_{i}}, \quad g_{i}=\left.e^{2 \sigma_{i}} g\right|_{U_{i}}
$$

From the definition it follows that any locally conformal coKähler manifold is normal. Also, one proves that for any $i, j \in I, i \neq j$, with $U_{i} \cap U_{j} \neq \emptyset$, one has $d \sigma_{i}=d \sigma_{j}$ on $U_{i} \cap U_{j}$, so that the 1 -forms $d \sigma_{i}$ glue up to a global closed 1-form $\theta$. Moreover, the Levi-Civita connections $\nabla^{i}$ of $\left(U_{i}, g_{i}\right)$ glue up to a globally defined torsion-free linear connection $D$ on $M$ given by

$$
D_{X} Y=\nabla_{X} Y-\frac{1}{2}(\theta(X) Y+\theta(Y) X-g(X, Y) B)
$$

where $B=\theta^{\sharp}$ and $\nabla$ is the Levi-Civita connection of $(M, g)$. It follows that $\mathrm{d} \eta=\eta \wedge \theta$ and $\mathrm{d} \Phi=-2 \Phi \wedge \theta$, $\Phi$ being the fundamental 2 -form of the almost contact metric manifold $(M, \phi, \xi, \eta, g)$. For more details we refer the reader to the aforementioned papers or also the monograph 62 .

We mention also the para-coKähler manifolds, studied, among others, by Dacko and Olszak (cf. [47, [53]). An almost paracontact structure (cf. [100]) on a $(2 n+1)$-dimensional smooth manifold $M$ is given by a $(1,1)$-tensor field $\tilde{\phi}$, a vector field $\xi$ and a 1 -form $\eta$ satisfying the following conditions

(i) $\eta(\xi)=1, \tilde{\phi}^{2}=I-\eta \otimes \xi$, 
(ii) the eigendistributions $\mathcal{D}^{+}$and $\mathcal{D}^{-}$of $\tilde{\phi}$ corresponding to the eigenvalues 1 and -1 , respectively, have equal dimension $n$.

An almost paracontact structure $(\tilde{\phi}, \xi, \eta)$ is said to be normal if the tensor field $N_{\tilde{\phi}}:=[\tilde{\phi}, \tilde{\phi}]-2 \mathrm{~d} \eta \otimes \xi$ vanishes identically. Equivalently, an almost paracontact manifold is normal if and only if the distributions $\mathcal{D}^{+}$and $\mathcal{D}^{-}$are integrable and $\xi$ is a foliated vector field with respect to both foliations ([28]). If an almost paracontact manifold is endowed with a semi-Riemannian metric $\tilde{g}$ such that

$$
\tilde{g}(\tilde{\phi} X, \tilde{\phi} Y)=-\tilde{g}(X, Y)+\eta(X) \eta(Y)
$$

for all $X, Y \in \Gamma(T M)$, then $(M, \tilde{\phi}, \xi, \eta, \tilde{g})$ is called an almost paracontact metric manifold. Notice that any such semi-Riemannian metric is necessarily of signature $(n, n+1)$, where the first index denotes the number of negative signs, and the above condition (ii) of the definition of almost paracontact structures is automatically satisfied. Moreover, as in the almost contact case, from (4.2) it follows easily that $\eta=\tilde{g}(\cdot, \xi)$ and $\tilde{g}(\cdot, \tilde{\phi} \cdot)=-\tilde{g}(\tilde{\phi} \cdot, \cdot)$. Hence one defines the fundamental 2 -form of the almost paracontact metric manifold by $\tilde{\Phi}(X, Y)=\tilde{g}(X, \tilde{\phi} Y)$. Now, if $\eta$ and $\tilde{\Phi}$ are closed the structure is said to be almost para-coKähler, and we point out that $(\eta, \tilde{\Phi})$ is then a cosymplectic structure. Finally, a normal almost para-coKähler manifold is said to be para-coKähler. Para-coKähler geometry has a long history, having its origins in the three papers [20, [21, 22] of Bouzon in the early 60's (even before than the Blair's definition of coKähler manifolds). The theory has some aspects similar to coKähler geometry even though there are also some relevant differences, for instance due to the non-positive definiteness of the metric.

Furthermore, another generalization of cosymplectic manifolds was proposed recently by de León, Merino, Oubiña, Rodrigues and Salgado in [57. A $k$-cosymplectic manifold is a smooth manifold $M$ of dimension $(k+1) n+k$ endowed with family $\left(\eta_{\alpha}, \omega_{\alpha}, \mathcal{F}\right)_{\alpha \in\{1, \ldots, k\}}$, where each $\eta_{\alpha}$ is a closed 1-form, each $\omega_{\alpha}$ is a closed 2 -form and $\mathcal{F}$ is a $n k$-dimensional foliation on $M$, related by the following conditions

(i) $\eta_{1} \wedge \cdots \wedge \eta_{k} \neq 0$,

(ii) $\eta_{\alpha}(X)=0$ and $\omega_{\alpha}\left(X, X^{\prime}\right)=0$ for any $X, X^{\prime} \in \Gamma(T \mathcal{F})$ and for each $\alpha \in\{1, \ldots, k\}$,

(iii) $\left(\bigcap_{\alpha=1}^{k} \operatorname{ker}\left(\eta_{\alpha}\right)\right) \cap\left(\bigcap_{\alpha=1}^{k} \operatorname{ker}\left(\omega_{\alpha}\right)\right)=\{0\}$ and $\operatorname{dim}\left(\bigcap_{\alpha=1}^{k} \operatorname{ker}\left(\omega_{\alpha}\right)\right)=k$.

Then on $M$ there are defined $k$ vector fields, $\xi_{1}, \ldots, \xi_{k}$, called Reeb vector fields, such that

$$
i_{\xi_{\alpha}} \eta_{\beta}=\delta_{\alpha \beta}, \quad i_{\xi_{\alpha}} \omega_{\beta}=0
$$

for any $\alpha, \beta \in\{1, \ldots, k\}$. The $k$-cosymplectic manifolds may be described locally in terms of Darboux coordinates $([57])$. In fact about any point of the manifold there are coordinates $\left(x_{\alpha}, y_{i}, z_{\alpha i}\right), 1 \leq \alpha \leq k$, $1 \leq i \leq n$, such that

$$
\eta_{\alpha}=d x_{\alpha}, \quad \omega_{\alpha}=\sum_{i=1}^{n} d y_{i} \wedge d z_{\alpha i}, \quad T \mathcal{F}=\operatorname{span}\left\{\frac{\partial}{\partial z_{11}}, \ldots, \frac{\partial}{\partial z_{k 1}}, \ldots, \frac{\partial}{\partial z_{1 n}}, \ldots, \frac{\partial}{\partial z_{k n}}\right\} .
$$

The $k$-cosymplectic structures were introduced in order to provide a suitable geometric setting for the description of classical field theories. The reader can find more information on the state of the art of the theory for instance in [122] or [133].

We conclude the subsection by mentioning the very recent concepts of "contact-symplectic pairs" and "generalized almost contact structure". A contact-symplectic pair of type $(h, k)$ on a smooth manifold $M$ of dimension $2 h+2 k+1$ is given by a 1 -form $\eta$ and a closed 2 -form $\omega$ such that $d \eta^{h+1}=0, \omega^{k+1}=0$ and $\eta \wedge d \eta^{h} \wedge \omega^{k}$ is a volume form, for some integers $h$ and $k$. For $k=0$ one retrieves the definition of contact structures and for $h=0$ that one of cosymplectic structures. This notion was introduced by Bande in [6], where he studies the main properties of such structures and finds examples on nilpotent Lie groups, nilmanifolds and principal torus bundles. 
Next, the theory of generalized almost contact manifolds is the attempt to extend to odd dimensions the very important theory of generalized complex structures, founded by Hitchin and Gualtieri (cf. 94, 92]). With this regard we mention the works of Iglesias-Ponte and Wade (96]), Vaisman ([148]), Poon and Wade ([131], [132]). In particular we recall the approach proposed by Vaisman and by Poon and Wade. According to their definition, a generalized almost contact structure on a smooth manifold $M$ consists of a bundle endomorphism $\phi: T M \oplus T^{*} M \longrightarrow T M \oplus T^{*} M$ and section $\xi+\eta$ of $T M \oplus T^{*} M$ such that

$$
\phi+\phi^{*}=0, \quad \phi^{2}=-I+\xi \odot \eta, \quad \eta(\xi)=1, \quad \phi(\xi)=\phi(\eta)=0 .
$$

Here the adjoint $\phi^{*}$ is with respect to the natural pairing of $T M \oplus T^{*} M$ defined by

$$
\langle X+\alpha, Y+\beta\rangle=\frac{1}{2}\left(i_{X} \beta+i_{Y} \alpha\right),
$$

and $\xi \odot \eta$ is the endomorphism defined by $\xi \odot \eta(X+\alpha)=\eta(X) \xi+\alpha(\xi) \eta$. This notion includes as particular cases both contact and almost cosymplectic structures. Moreover, Poon and Wade discuss possible notions of normality / integrability.

4.4. Almost coKähler manifolds satisfying further conditions. Another important topic in coKähler geometry is given by the study of some classes of almost coKähler manifolds satisfying certain additional conditions (for instance concerning the curvature tensor). More generally, one considers some sub-classes of almost contact metric manifolds which satisfy conditions closely related to the coKähler manifolds. Probably the most important example is given by the nearly coKähler manifolds. An almost contact metric manifold $(M, \phi, \xi, \eta, g)$ is called nearly coKähler if the structure tensor $\phi$ and the 1-form $\eta$ are Killing, that is

$$
\left(\nabla_{X} \phi\right) Y+\left(\nabla_{Y} \phi\right) X=0, \quad\left(\nabla_{X} \eta\right)(Y)+\left(\nabla_{Y} \eta\right)(X)=0
$$

for any $X, Y \in \Gamma(T M)$. The second condition in (4.3), which is equivalent to requiring that $\xi$ is Killing, is usually included in the definition, but however it is a consequence of the first one (cf. [15, Proposition 6.1]). The name "nearly coKähler" is due to the property that a normal nearly coKähler manifold is necessarily coKähler ([13]). Moreover, it is known that any 3-dimensional nearly coKähler manifold is coKähler (99). A well-known nearly coKähler manifold is $S^{5}$ endowed with the almost contact metric structure induced by the almost Hermitian structure of $S^{6}$ defined by means of the cross product of the imaginary part of the octonions (see, for more details, [15] page 63 and 102). This gives an example of non-normal almost contact metric structure on an odd-dimensional sphere. Nearly coKähler manifolds, in particular their curvature and topological properties, have been studied by several authors (see e.g. [103, [5, 70], 82, 71]).

Another interesting topic concerns the study of almost contact metric manifolds admitting a Weyl structure. Recall that a Weyl structure on a manifold $M$ of dimension $m \geq 3$ is defined by a pair $W=\left([g], D^{[g]}\right)$, where $[g]$ is a conformal class of Riemannian metrics and $D^{[g]}$ is the unique torsion-free connection, called Weyl connection, satisfying

$$
D^{[g]} g=-2 \theta \otimes g .
$$

for some 1-form $\theta$. The Weyl structure is said to be closed if $\mathrm{d} \theta=0$ and Einstein-Weyl if there exists a smooth function $\Lambda$ on $M$ such that

$$
\operatorname{Ric}^{D^{[g]}}(X, Y)+\operatorname{Ric}^{D^{[g]}}(Y, X)=\Lambda g(X, Y),
$$

where $\operatorname{Ric}^{D^{[g]}}$ denotes the Ricci tensor with respect to the connection $D^{[g]}$. Since the condition (4.4) is invariant under Weyl transformations $g^{\prime}=e^{2 f} g, \theta^{\prime}=\theta+\mathrm{d} f$, with $f \in C^{\infty}(M)$, one sometimes abuses the terminology by choosing a Riemannian metric in $[g]$ and referring to the pair $W=(g, \theta)$ as a Weyl structure. The existence of Einstein-Weyl structures on almost contact metric manifolds has 
been recently investigated by several authors. Concerning coKähler geometry, we mention the result that locally conformal coKähler manifolds admit a naturally defined conformally invariant Weyl structure ([119]). Later on, Matzeu proved that every $(2 n+1)$-dimensional coKähler manifold $(M, \phi, \xi, \eta, g)$ of constant $\phi$-sectional curvature $c>0$ admits two Ricci-flat Weyl structures where the 1-forms associated to the metric $g \in[g]$ are $\pm \theta= \pm \lambda \eta$, where $\lambda=\frac{2 c}{2 n-1}$. More recently, she generalized such a result by proving that if a compact coKähler manifold $(M, \phi, \xi, \eta, g)$ admits a closed Einstein-Weyl structure $D^{[g]}$, then $M$ is necessarily $\eta$-Einstein $([120])$.

A further well-known class of almost coKähler manifolds satisfying some remarkable curvature condition is that of conformally flat almost coKähler manifolds. In dimension greater than or equal to 5 those manifolds are of non-positive scalar curvature and are coKähler if and only if they are locally flat (cf. [125, [127]). Moreover, again under the assumption $\operatorname{dim}(M) \geq 5$, any conformally flat almost coKähler manifold with Kählerian leaves is necessarily coKähler ([50]). Contrary to that, in dimension 3, there are examples of conformally fiat almost coKähler manifolds (which necessarily have Kählerian leaves) that are not locally fiat and not coKähler (see again [50] and [48]).

\section{REFERENCES}

[1] C. Albert, Le théorème de réduction de Marsden-Weinstein en géométrie cosymplectique et de contact, J. Geom. Phys. 6 (1989), no. 4, 627-649.

[2] P. Alegre, D. E. Blair, and A. Carriazo, Generalized Sasakian-space-forms, Israel J. Math. 141 (2004), $157-183$.

[3] J. M. Arms, J. E. Marsden, and V. Moncrief, Symmetry and bifurcations of momentum mappings, Comm. Math. Phys. 78 (1980/81), no. 4, 455-478.

[4] L. Auslander, L. Green, and F. Hahn, Flows on homogeneous spaces, With the assistance of L. Markus and W. Massey, and an appendix by L. Greenberg. Annals of Mathematics Studies, No. 53, Princeton University Press, Princeton, N.J., 1963.

[5] M. Banaru, On nearly-cosymplectic hypersurfaces in nearly-Kählerian manifolds, Studia Univ. Babeş-Bolyai Math. 47 (2002), no. 3, 3-11.

[6] Gianluca Bande, Couples contacto-symplectiques, Trans. Amer. Math. Soc. 355 (2003), no. 4, 1699-1711 (electronic).

[7] M. Barbero-Liñán, A. Echeverría-Enríquez, D. Martín de Diego, M. C. Muñoz-Lecanda, and N. Román-Roy, Unified formalism for nonautonomous mechanical systems, J. Math. Phys. 49 (2008), no. 6, 062902, 14.

[8] G. Bazzoni, M. Fernández, and V. Muñoz, Non-formal co-symplectic manifolds, ArXiv e-prints (2012), arXiv:1203.6422.

[9] G. Bazzoni and J. Oprea, On the structure of cokähler manifolds, to appear in Geom. Dedicata, DOI:10.1007/s10711013-9869-7 (2013).

[10] A. Bejancu, Geometry of CR-submanifolds, Mathematics and its Applications (East European Series), vol. 23, D. Reidel Publishing Co., Dordrecht, 1986.

[11] A. Bejancu and D. Smaranda, Semi-invariant submanifolds of a co-Kähler manifold, An. Ştiinţ. Univ. Al. I. Cuza Iaşi Secț. I a Mat. 29 (1983), no. 2, suppl., 27-32.

[12] D. E. Blair, The theory of quasi-Sasakian structures, J. Differential Geometry 1 (1967), 331-345.

[13] _ Almost contact manifolds with Killing structure tensors, Pacific J. Math. 39 (1971), 285-292.

[14] _ Contact manifolds in Riemannian geometry, Lecture Notes in Mathematics, Vol. 509, Springer-Verlag, Berlin, 1976.

[15] _ Riemannian geometry of contact and symplectic manifolds, second ed., Progress in Mathematics, vol. 203, Birkhäuser Boston Inc., Boston, MA, 2010.

[16] D. E. Blair and S. I. Goldberg, Topology of almost contact manifolds, J. Differential Geometry 1 (1967), $347-354$.

[17] D. E. Blair, T. Koufogiorgos, and Basil J. Papantoniou, Contact metric manifolds satisfying a nullity condition, Israel J. Math. 91 (1995), no. 1-3, 189-214.

[18] E. Boeckx, A full classification of contact metric ( $k, \mu)$-spaces, Illinois J. Math. 44 (2000), no. 1, 212-219.

[19] E. Boeckx and C. Gherghe, Harmonic maps and cosymplectic manifolds, J. Aust. Math. Soc. 76 (2004), no. 1, 75-92.

[20] J. Bouzon, Structures presque cocomplexes. I, C. R. Acad. Sci. Paris 255 (1962), 822-824.

[21] — Structures presque cocomplexes. II, C. R. Acad. Sci. Paris 255 (1962), 1061-1063.

[22] Structures presque-cohermitiennes, C. R. Acad. Sci. Paris 258 (1964), 412-415.

[23] C. P. Boyer and K. Galicki, Sasakian geometry, Oxford Mathematical Monographs, Oxford University Press, Oxford, 2008. 
[24] M. L. Bruschi, Un théorème de décomposition pour les variétés cosymplectiques homogènes, C. R. Acad. Sci. Paris 257 (1963), 4120-4121.

[25] J. Brylinski, A differential complex for Poisson manifolds, J. Differential Geom. 28 (1988), no. 1, 93-114.

[26] F. Cantrijn and J. Cortés, Cosymplectic reduction of constrained systems with symmetry, Rep. Math. Phys. 49 (2002), no. 2-3, 167-182, XXXIII Symposium on Mathematical Physics (Torún, 2001).

[27] F. Cantrijn, M. de León, and E. A. Lacomba, Gradient vector fields on cosymplectic manifolds, J. Phys. A 25 (1992), no. $1,175-188$.

[28] B. Cappelletti-Montano, Bi-Legendrian structures and paracontact geometry, Int. J. Geom. Methods Mod. Phys. 6 (2009), no. 3, 487-504.

[29] , The foliated structure of contact metric $(\kappa, \mu)$-spaces, Illinois J. Math. 53 (2009), no. 4, 1157-1172.

[30] B. Cappelletti-Montano and A. De Nicola, 3-Sasakian manifolds, 3-cosymplectic manifolds and Darboux theorem, J. Geom. Phys. 57 (2007), no. 12, 2509-2520.

[31] B. Cappelletti-Montano, A. De Nicola, and G. Dileo, 3-quasi-Sasakian manifolds, Ann. Global Anal. Geom. 33 (2008), no. 4, 397-409.

[32] _ The geometry of 3-quasi-Sasakian manifolds, Internat. J. Math. 20 (2009), no. 9, 1081-1105.

[33] B. Cappelletti-Montano, A. De Nicola, and I. Yudin, Topology of 3-cosymplectic manifolds, Q. J. Math. 64 (2013), no. 1, 59-82.

[34] B. Cappelletti-Montano and A. M. Pastore, Einstein-like conditions and cosymplectic geometry, J. Adv. Math. Stud. 3 (2010), no. 2, 27-40.

[35] M. Capursi, Some remarks on the product of two almost contact manifolds, An. Ştiinţ. Univ. Al. I. Cuza Iaşi Secţ. I a Mat. 30 (1984), no. 1, 75-79.

[36] Alfonso Carriazo and Verónica Martín-Molina, Almost cosymplectic and almost kenmotsu $(\kappa, \mu, \nu)$-spaces, Mediterranean Journal of Mathematics 10 (2013), no. 3, 1551-1571 (English).

[37] D. Chinea, Harmonicity on maps between almost contact metric manifolds, Acta Math. Hungar. 126 (2010), no. 4, $352-365$.

[38] D. Chinea, M. de León, and J. C. Marrero, Locally conformal cosymplectic manifolds and time-dependent Hamiltonian systems, Comment. Math. Univ. Carolin. 32 (1991), no. 2, 383-387.

[39] _ Stability of invariant foliations on almost contact manifolds, Publ. Math. Debrecen 43 (1993), no. 1-2, 41-52.

[40] _ Topology of cosymplectic manifolds, J. Math. Pures Appl. (9) 72 (1993), no. 6, 567-591.

[41] _ Coeffective cohomology on almost cosymplectic manifolds, Bull. Sci. Math. 119 (1995), no. 1, 3-20.

[42] D. Chinea and C. González, An example of an almost cosymplectic homogeneous manifold, Differential geometry, Peñíscola 1985, Lecture Notes in Math., vol. 1209, Springer, Berlin, 1986, pp. 133-142.

[43] — A classification of almost contact metric manifolds, Ann. Mat. Pura Appl. (4) 156 (1990), 15-36.

[44] L. A. Cordero, M. Fernández, and M. de León, Examples of compact almost contact manifolds admitting neither Sasakian nor cosymplectic structures, Atti Sem. Mat. Fis. Univ. Modena 34 (1985/86), no. 1, 43-54.

[45] P. Dacko, Note on left invariant almost cosymplectic structures, Period. Math. Hungar. 38 (1999), no. 3, $167-171$.

[46] On almost cosymplectic manifolds with the structure vector field $\xi$ belonging to the $k$-nullity distribution, Balkan J. Geom. Appl. 5 (2000), no. 2, 47-60.

[47] _ On almost para-cosymplectic manifolds, Tsukuba J. Math. 28 (2004), no. 1, 193-213.

[48] _ On three dimensional conformally flat almost cosymplectic manifolds, ArXiv e-prints (2007), arXiv:0710.1042.

[49] — On CR (Cauchy-Riemann) almost cosymplectic manifolds, ArXiv e-prints (2012), arXiv:1209.1848.

[50] P. Dacko and Z. Olszak, On conformally flat almost cosymplectic manifolds with Kählerian leaves, Rend. Sem. Mat. Univ. Politec. Torino 56 (1998), no. 1, 89-103.

[51] _ On almost cosymplectic $(-1, \mu, 0)$-spaces, Cent. Eur. J. Math. 3 (2005), no. 2, 318-330 (electronic).

[52] On almost cosymplectic $(\kappa, \mu, \nu)$-spaces, PDEs, submanifolds and affine differential geometry, Banach Center Publ., vol. 69, Polish Acad. Sci., Warsaw, 2005, pp. 211-220.

[53] _ On weakly para-cosymplectic manifolds of dimension 3, J. Geom. Phys. 57 (2007), no. 2, 561-570.

[54] M. de León, J. Marín-Solano, and J. C. Marrero, The constraint algorithm in the jet formalism, Differential Geom. Appl. 6 (1996), no. 3, 275-300.

[55] M. de León and J. C. Marrero, Constrained time-dependent Lagrangian systems and Lagrangian submanifolds, J. Math. Phys. 34 (1993), no. 2, 622-644.

[56] _ Compact cosymplectic manifolds with transversally positive definite Ricci tensor, Rend. Mat. Appl. (7) 17 (1997), no. 4, 607-624.

[57] M. de León, E. Merino, J. A. Oubiña, P. R. Rodrigues, and M. Salgado, Hamiltonian systems on k-cosymplectic manifolds, J. Math. Phys. 39 (1998), no. 2, 876-893.

[58] M. de León and P. R. Rodrigues, Methods of differential geometry in analytical mechanics, North-Holland Mathematics Studies, vol. 158, North-Holland Publishing Co., Amsterdam, 1989. 
[59] M. de León and M. Saralegi, Cosymplectic reduction for singular momentum maps, J. Phys. A 26 (1993), no. 19, 5033-5043.

[60] M. de León and G. M. Tuynman, A universal model for cosymplectic manifolds, J. Geom. Phys. 20 (1996), no. 1, $77-86$.

[61] P. Deligne, P. Griffiths, J. Morgan, and D. Sullivan, Real homotopy theory of Kähler manifolds, Invent. Math. 29 (1975), no. 3, 245-274.

[62] S. Dragomir and L. Ornea, Locally conformal Kähler geometry, Progress in Mathematics, vol. 155, Birkhäuser Boston Inc., Boston, MA, 1998.

[63] S. Dragomir and D. Perrone, Harmonic vector fields : variational principles and differential geometry, Elsevier, 2012.

[64] A. Echeverría Enríquez, M. C. Muñoz Lecanda, and N. Román-Roy, Geometrical setting of time-dependent regular systems. Alternative models, Rev. Math. Phys. 3 (1991), no. 3, 301-330.

[65] H. Endo, Remarks on invariant submanifolds in an almost cosymplectic manifold, Tensor (N.S.) 42 (1985), no. 2, $165-167$.

[66] _ On invariant submanifolds in almost cosymplectic manifolds, Colloq. Math. 57 (1989), no. 1, 69-72.

[67] _ On Ricci curvatures of almost cosymplectic manifolds, An. Ştiinţ. Univ. Al. I. Cuza Iaşi Secţ. I a Mat. 40 (1994), no. 1, 75-83.

[68] On the AC-contact Bochner curvature tensor field on almost cosymplectic manifolds, Publ. Inst. Math. (Beograd) (N.S.) 56(70) (1994), 102-110.

[69] , Non-existence of almost cosymplectic manifolds satisfying a certain condition, Tensor (N.S.) 63 (2002), no. 3, $272-284$.

[70] - On the curvature tensor of nearly cosymplectic manifolds of constant $\Phi$-sectional curvature, An. Ştiinţ. Univ. Al. I. Cuza Iaşi. Mat. (N.S.) 51 (2005), no. 2, 439-454.

[71] _ On the first Betti number of certain compact nearly cosymplectic manifolds, J. Geom. 103 (2012), no. 2, 231-236.

[72] S. Eum, Cosymplectic manifolds of constant $\phi$-holomorphic curvature, Bull. Korean Math. Soc. 9 (1972), 1-7.

[73] M. Falcitelli, S. Ianus, and A. M. Pastore, Riemannian submersions and related topics, World Scientific Publishing Co. Inc., River Edge, NJ, 2004.

[74] Y. Félix, J. Oprea, and D. Tanré, Algebraic models in geometry, Oxford Graduate Texts in Mathematics, vol. 17, Oxford University Press, Oxford, 2008.

[75] M. Fernández and A. Gray, Compact symplectic solvmanifolds not admitting complex structures, Geom. Dedicata 34 (1990), no. 3, 295-299.

[76] M. Fernández, R. Ibáñez, and M. de León, A Nomizu's theorem for the coeffective cohomology, Math. Z. 226 (1997), no. $1,11-23$.

[77] _ The canonical spectral sequences for Poisson manifolds, Israel J. Math. 106 (1998), 133-155.

[78] Coeffective and de Rham cohomologies on almost contact manifolds, Differential Geom. Appl. 8 (1998), no. 3, 285-303.

[79] D. Fetcu, Maps between almost Kähler manifolds and framed $\phi$-manifolds, Balkan J. Geom. Appl. 9 (2004), no. 2, 13-24.

[80] D. Fetcu and H. Rosenberg, Surfaces with parallel mean curvature in $\mathbb{C} P^{n} \times \mathbb{R}$ and $\mathbb{C} H^{n} \times \mathbb{R}$, Trans. Amer. Math. Soc. 366 (2014), 75-94.

[81] A. Fino and L. Vezzoni, Some results on cosymplectic manifolds, Geom. Dedicata 151 (2011), 41-58.

[82] S. Fueki and H. Endo, On conformally flat nearly cosymplectic manifolds, Tensor (N.S.) 66 (2005), no. 3, 305-316.

[83] A. Fujimoto and H. Mutō, On cosymplectic manifolds, Tensor (N.S.) 28 (1974), 43-52.

[84] C. Gherghe, Harmonic morphisms on some almost contact metric manifolds, Tensor (N.S.) 61 (1999), no. 3, $276-281$.

[85] _ Harmonicity on cosymplectic manifolds, Rocky Mountain J. Math. 40 (2010), no. 6, 1875-1885.

[86] E. Ghys and V. Sergiescu, Stabilité et conjugaison différentiable pour certains feuilletages, Topology 19 (1980), no. 2 , 179-197.

[87] S. I. Goldberg, Curvature and homology, Pure and Applied Mathematics, Vol. XI, Academic Press, New York, 1962.

[88] _ Totally geodesic hypersurfaces of Kaehler manifolds, Pacific J. Math. 27 (1968), 275-281.

[89] _ Invariant submanifolds of codimension 2 of almost contact manifolds, Ann. Scuola Norm. Sup. Pisa (3) 25 (1971), 377-388.

[90] S. I. Goldberg and K. Yano, Integrability of almost cosymplectic structures, Pacific J. Math. 31 (1969), 373-382.

[91] M. J. Gotay and G. M. Tuynman, $\mathbf{R}^{2 n}$ is a universal symplectic manifold for reduction, Lett. Math. Phys. 18 (1989), no. $1,55-59$.

[92] M. Gualtieri, Generalized complex geometry, Ann. of Math. (2) 174 (2011), no. 1, 75-123.

[93] E. Guzmán and J. C. Marrero, Time-dependent mechanics and Lagrangian submanifolds of presymplectic and Poisson manifolds, J. Phys. A 43 (2010), no. 50, 505201, 23.

[94] N. Hitchin, Generalized Calabi-Yau manifolds, Q. J. Math. 54 (2003), no. 3, 281-308. 
[95] C. Hummel, Gromov's compactness theorem for pseudo-holomorphic curves, Progress in Mathematics, vol. 151, Birkhäuser Verlag, Basel, 1997.

[96] D. Iglesias-Ponte and A. Wade, Contact manifolds and generalized complex structures, J. Geom. Phys. 53 (2005), no. 3, 249-258.

[97] S. Ishihara, Quaternion Kählerian manifolds and fibred Riemannian spaces with Sasakian 3-structure, Kōdai Math. Sem. Rep. 25 (1973), 321-329.

[98] D. Janssens and L. Vanhecke, Almost contact structures and curvature tensors, Kodai Math. J. 4 (1981), no. 1, 1-27.

[99] J. B. Jun, I. B. Kim, and U. K. Kim, On 3-dimensional almost contact metric manifolds, Kyungpook Math. J. 34 (1994), no. 2, 293-301.

[100] S. Kaneyuki and F. L. Williams, Almost paracontact and parahodge structures on manifolds, Nagoya Math. J. 99 (1985), 173-187.

[101] J. Kim, J. Choi, and M. M. Tripathi, On generic submanifolds of manifolds equipped with a hypercosymplectic 3structure, Commun. Korean Math. Soc. 21 (2006), no. 2, 321-335.

[102] U. K. Kim, On anti-invariant submanifolds of cosymplectic manifolds, J. Korean Math. Soc. 20 (1983), no. 1, 9-29.

[103] V. F. Kiritchenko, Sur la géométrie des variétés approximativement cosymplectiques, C. R. Acad. Sci. Paris Sér. I Math. 295 (1982), no. 12, 673-676.

[104] J. Koszul, Crochet de Schouten-Nijenhuis et cohomologie, Astérisque (1985), no. Numero Hors Serie, 257-271, The mathematical heritage of Élie Cartan (Lyon, 1984).

[105] Y. Kuo, On almost contact 3-structure, Tôhoku Math. J. (2) 22 (1970), 325-332.

[106] I. Lacirasella, J. C. Marrero, and E. Padrón, Reduction of symplectic principal $\mathbb{R}$-bundles, J. Phys. A 45 (2012), no. $32,325202,29$

[107] H. Li, Topology of co-symplectic/co-Kähler manifolds, Asian J. Math. 12 (2008), no. 4, 527-543.

[108] P. Libermann, Sur les automorphismes infinitésimaux des structures symplectiques et des structures de contact, Colloque Géom. Diff. Globale (Bruxelles, 1958), Centre Belge Rech. Math., Louvain, 1959, pp. 37-59.

[109] Sur quelques exemples de structures pfaffiennes et presque cosymplectiques, Ann. Mat. Pura Appl. (4) 60 (1962), 153-172.

[110] P. Libermann and C. M. Marle, Symplectic geometry and analytical mechanics, Mathematics and its Applications, vol. 35, D. Reidel Publishing Co., Dordrecht, 1987, Translated from the French by Bertram Eugene Schwarzbach.

[111] A. Lichnerowicz, Sur la réductivité de certaines algèbres d'automorphismes, C. R. Acad. Sci. Paris 253 (1961), 1302-1304.

[112] — Théorèmes de réductivité sur des algèbres d'automorphismes, Rend. Mat. e Appl. (5) 22 (1963), $197-244$.

[113] G. D. Ludden, Submanifolds of cosymplectic manifolds, J. Differential Geometry 4 (1970), 237-244.

[114] J. C. Marrero, Some curvature properties of locally conformal co-Kähler manifolds, Rev. Acad. Canaria Cienc. 1 (1990), 261-270.

[115] J. C. Marrero and E. Padrón, New examples of compact cosymplectic solvmanifolds, Arch. Math. (Brno) 34 (1998), no. $3,337-345$.

[116] J. Marsden and A. Weinstein, Reduction of symplectic manifolds with symmetry, Rep. Mathematical Phys. 5 (1974), no. $1,121-130$.

[117] J. E. Marsden and T. Ratiu, Reduction of Poisson manifolds, Lett. Math. Phys. 11 (1986), no. 2, 161-169.

[118] F. Martín Cabrera, Almost hyper-Hermitian structures in bundle spaces over manifolds with almost contact 3structure, Czechoslovak Math. J. 48(123) (1998), no. 3, 545-563.

[119] P. Matzeu, Almost contact Einstein-Weyl structures, Manuscripta Math. 108 (2002), no. 3, 275-288.

[120] _ Closed Einstein-Weyl structures on compact Sasakian and cosymplectic manifolds, Proc. Edinb. Math. Soc. (2) 54 (2011), no. 1, 149-160.

[121] A. Morimoto, On normal almost contact structures, J. Math. Soc. Japan 15 (1963), 420-436.

[122] M. C. Muñoz-Lecanda, M. Salgado, and S. Vilariño, k-symplectic and $k$-cosymplectic Lagrangian field theories: some interesting examples and applications, Int. J. Geom. Methods Mod. Phys. 7 (2010), no. 4, 669-692.

[123] K. Ogiue, G-structures definied by tensor fields, Kōdai Math. Sem. Rep. 20 (1968), 54-75.

[124] M. Okumura, Cosymplectic hypersurfaces in Kaehlerian manifold of constant holomorphic sectional curvature, Kōdai Math. Sem. Rep. 17 (1965), 63-73.

[125] Z. Olszak, On almost cosymplectic manifolds, Kodai Math. J. 4 (1981), no. 2, 239-250.

[126] _ Almost cosymplectic real hypersurfaces in Kähler manifolds, Arch. Math. (Brno) 18 (1982), no. 4, $187-192$.

[127] — Almost cosymplectic manifolds with kählerian leaves, Tensor N. S. 46 (1987), 117-124.

[128] _ Locally conformal almost cosymplectic manifolds, Colloq. Math. 57 (1989), no. 1, 73-87.

[129] H. K. Pak and T. W. Kim, Almost cosymplectic manifolds admitting harmonic characteristic vector fields, Adv. Stud. Contemp. Math. (Kyungshang) 13 (2006), no. 1, 75-80.

[130] D. Perrone, Classification of homogeneous almost cosymplectic three-manifolds, Differential Geom. Appl. 30 (2012), no. $1,49-58$. 
[131] Y. S. Poon and A. Wade, Approaches to generalize contact structures, Pure Appl. Math. Q. 6 (2010), no. 2, Special Issue: In honor of Michael Atiyah and Isadore Singer, 603-622.

[132] _ Generalized contact structures, J. Lond. Math. Soc. (2) 83 (2011), no. 2, 333-352.

[133] A. M. Rey, N. Román-Roy, M. Salgado, and S. Vilariño, $k$-cosymplectic classical field theories: Tulczyjew and SkinnerRusk formulations, Math. Phys. Anal. Geom. 15 (2012), no. 2, 85-119.

[134] S. Sasaki, On differentiable manifolds with certain structures which are closely related to almost contact structure. I, Tôhoku Math. J. (2) 12 (1960), 459-476.

[135] S. Sasaki and Y. Hatakeyama, On differentiable manifolds with certain structures which are closely related to almost contact structure. II, Tôhoku Math. J. (2) 13 (1961), 281-294.

[136] D. J. Saunders, The geometry of jet bundles, London Mathematical Society Lecture Note Series, vol. 142, Cambridge University Press, Cambridge, 1989.

[137] R. Sjamaar and E. Lerman, Stratified symplectic spaces and reduction, Ann. of Math. (2) 134 (1991), no. 2, 375-422.

[138] Y. Song, J. Kim, and M. M. Tripathi, On hypersurfaces of manifolds equipped with a hypercosymplectic 3-structure, Commun. Korean Math. Soc. 18 (2003), no. 2, 297-308.

[139] D. Sullivan, Infinitesimal computations in topology, Inst. Hautes Études Sci. Publ. Math. (1977), no. 47, $269-331$.

[140] S. Takizawa, On contact structures of real and complex manifolds, Tôhoku Math. J. (2) 15 (1963), $227-252$.

[141] Y. Tashiro, On contact structure of hypersurfaces in complex manifolds. I, Tôhoku Math. J. (2) 15 (1963), 62-78.

[142] - On contact structure of hypersurfaces in complex manifolds. II, Tôhoku Math. J. (2) 15 (1963), $167-175$.

[143] D. Tischler, On fibering certain foliated manifolds over $S^{1}$, Topology 9 (1970), 153-154.

[144] F. Tricerri and L. Vanhecke, Homogeneous structures on Riemannian manifolds, London Mathematical Society Lecture Note Series, vol. 83, Cambridge University Press, Cambridge, 1983.

[145] W. M. Tulczyjew, Les sous-variétés lagrangiennes et la dynamique hamiltonienne, C. R. Acad. Sci. Paris Sér. A-B 283 (1976), no. 1, Ai, A15-A18.

[146] W. M. Tulczyjew and P. Urbański, Liouville structures, Univ. Iagel. Acta Math. (2009), no. 47, 187-226.

[147] C. Udrişte, Structures presque coquaternioniennes, Bull. Math. Soc. Sci. Math. R. S. Roumanie (N.S.) 13(61) (1969), no. 4, 487-507.

[148] I. Vaisman, Generalized CRF-structures, Geom. Dedicata 133 (2008), 129-154.

[149] H. Wakakuwa, On Riemannian manifolds with homogeneous holonomy group $\operatorname{Sp}(n)$, Tôhoku Math. J. (2) 10 (1958), 274-303.

[150] B. Watson, New examples of strictly almost Kähler manifolds, Proc. Amer. Math. Soc. 88 (1983), no. 3, 541-544.

[151] K. Yano and M. Kon, CR submanifolds of Kaehlerian and Sasakian manifolds, Progress in Mathematics, vol. 30, Birkhäuser Boston, Mass., 1983.

Dipartimento di Matematica e Informatica, Università degli Studi di Cagliari, Via Ospedale 72,09124 CAgLiari

E-mail address: b.cappellettimontano@gmail.com

CMuC, Department of Mathematics, University of Coimbra, Apartado 3008, 3001-454 Coimbra, Portugal E-mail address: antondenicola@gmail.com

CMuC, Department of Mathematics, University of Coimbra, Apartado 3008, 3001-454 Coimbra, Portugal E-mail address: yudin@mat.uc.pt 